\title{
Non-equilibrium steady states in the Klein-Gordon theory
}

\author{
Benjamin Doyon ${ }^{1, *}$, Andrew Lucas ${ }^{2}$, \\ Koenraad Schalm ${ }^{2,3}$ and M. J. Bhaseen ${ }^{4}$ \\ ${ }^{1}$ Department of Mathematics, King's College London, Strand, London, U.K. \\ 2 Department of Physics, Harvard University, Cambridge, MA 02138 USA. \\ ${ }^{3}$ Instituut-Lorentz, Leiden University, Leiden, The Netherlands. \\ ${ }^{4}$ Department of Physics, King's College London, Strand, London, U.K. \\ * email: benjamin.doyon@kcl.ac.uk
}

\begin{abstract}
We construct non-equilibrium steady states in the Klein-Gordon theory in arbitrary space dimension $d$ following a local quench. We consider the approach where two independently thermalized semi-infinite systems, with temperatures $T_{\mathrm{L}}$ and $T_{\mathrm{R}}$, are connected along a $d$-1-dimensional hypersurface. A current-carrying steady state, described by thermally distributed modes with temperatures $T_{\mathrm{L}}$ and $T_{\mathrm{R}}$ for left and right-moving modes, respectively, emerges at late times. The non-equilibrium density matrix is the exponential of a non-local conserved charge. We obtain exact results for the average energy current and the complete distribution of energy current fluctuations. The latter shows that the long-time energy transfer can be described by a continuum of independent Poisson processes, for which we provide the exact weights. We further describe the full time evolution of local observables following the quench. Averages of generic local observables, including the stress-energy tensor, approach the steady state with a power-law in time, where the exponent depends on the initial conditions at the connection hypersurface. We describe boundary conditions and special operators for which the steady state is reached instantaneously on the connection hypersurface. A semiclassical analysis of freely propagating modes yields the average energy current at large distances and late times. We conclude by comparing and contrasting our findings with results for interacting theories and provide an estimate for the timescale governing the crossover to hydrodynamics. As a modification of our Klein-Gordon analysis we also include exact results for free Dirac fermions.
\end{abstract}




\section{Contents}

1 Introduction 2

2 Exact steady-state density matrix, averages and fluctuations 5

2.1 The exact steady-state density matrix $\ldots \ldots \ldots \ldots \ldots \ldots$

2.2 Steady-state averages of the energy current and energy density . . . . . . 8

2.3 Exact cumulant generating function of the energy transfer . . . . . . . . . . 9

2.4 Non-locality of the steady-state density matrix and power-law correlations . . . . 12

3 Exact time evolution and the steady state density matrix 14

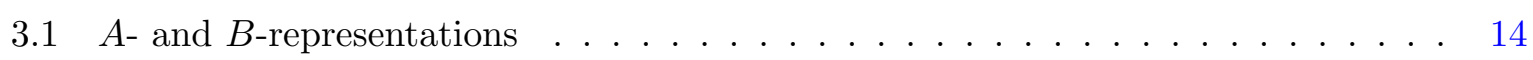

3.2 Time-evolved operators: main results . . . . . . . . . . . . . . 15

3.3 Long-time limit and approach to the steady state . . . . . . . . . . . . . 17

3.4 Time-evolution of the average energy current and energy density . . . . . . . . . 19

3.5 Semiclassical analysis of energy flow evolution and transition regions . . . . . . 20

4 Discussion: effects of interactions 23

5 Conclusion 24

$\begin{array}{ll}\text { Acknowledgements } & 25\end{array}$

$\begin{array}{ll}\text { A Energy current and energy density } & 25\end{array}$

B Steady-state density matrix in terms of local fields $\quad 26$

$\begin{array}{ll}\text { C Time evolution of fields } & 27\end{array}$

D Asymptotic time evolution of the energy current 30

E Numerical simulations $\quad 34$

F Correlation functions in the steady state $\quad 35$

G Dimensional reduction $\quad 36$ 


\section{Introduction}

Understanding far-from-equilibrium phenomena is one of the most important challenges of current theoretical physics research. Amongst these phenomena, non-equilibrium steady states (NESS), involving constant flows of energy, particles or charge between leads, play an important role in both theory and experiment. Although stationary, they exhibit many of the non-trivial features associated with non-equilibrium physics, including generalized fluctuation relations $[1,2,3]$. The study of quantum NESS is of particular interest, especially in the presence of emergent collective behavior, as it sheds light on the interplay between quantum effects and non-equilibrium physics.

From a theoretical perspective, there are a variety of ways to represent NESS. Here, we consider the partitioning approach, or Hamiltonian-reservoir formulation, where the baths are fully and exactly represented. This is a real-time construction of NESS, whereby two infinitely long leads are initially thermalized in different equilibrium states, and are then suddenly connected (either to another quantum system, or just to each other) and allowed to evolve unitarily for a long time. Such constructions have been used in a variety of different contexts. For example, in combination with Keldysh perturbation theory, they have been used in order to study charge currents through nanostructures [4]. They have also been used to study thermal flows in infinite classical chains of harmonic oscillators [5, 6]. Quantum transport has also been investigated within the $C^{*}$-algebra formalism for the free-fermionic $[7,8]$ and XY $[9,10,11]$ quantum chains; for general results see $[12,13]$. In modern parlance within the physics community, this formulation is a "local quench" connecting initial mixed states.

The Hamiltonian-reservoir formulation is particularly well adapted to the study of the interplay between quantum collective behavior and non-equilibrium physics, as it is readily formulated within quantum field theory (QFT). In the context of quantum impurity models, where the leads are described by fermionic seas with free gapless excitations, this point of view has been very successful. Various aspects of perturbation theory $[14,15,16,17,18,19,20]$, integrability [21, 22, 23, 24, 25] and non-equilibrium fluctuation theory [26, 27, 28, 29, 24, 25, 30] have been developed. More recently, attention has focused on models where the leads exhibit non-trivial emergent properties. Non-equilibrium flows, including the full fluctuation theory, have been studied in homogeneous critical systems described by conformal field theory (CFT) $[31,32,33,34]$, in universal regions described by integrable QFT [35, 36], and in the quantum Ising model [37]. The CFT predictions have been confirmed numerically using time-dependent density matrix renormalization group simulations of quantum spin chains [38, 39].

In all of these examples, the system considered is one-dimensional. The generalization to $d$-dimensional leads connected along a $d-1$ dimensional hypersurface is quite non-trivial due to the absence of widely applicable exact techniques. Recent progress on this problem was achieved for strongly interacting quantum critical models [40] using a combination of insights 
derived from QFT, gauge-gravity duality and fluid dynamics. It was shown that the NESS for thermal transport in these interacting quantum systems is fully described by a Lorentz boosted thermal state.

In this work, we focus on the opposite limit of vanishing interactions, by considering the free massive Klein-Gordon theory in $d$-dimensions, with $d \geq 1$. The complete time-evolution, including the emergence of a NESS, is fully amenable to theoretical treatment. This model describes the low-energy scaling limit of an array of coupled harmonic oscillators, and as such provides a paradigmatic model for studying thermal transport in detail. After the original works $[41,6,42]$ in the classical realm, quantum harmonic crystals have been studied within various formalisms [44, 45, 46, 47, 48, 49, 50]. This also includes studies in higher dimensions $[42,43,49]$, with the restriction that the transverse directions be finite. Here, we consider the far from equilibrium response of the genuinely infinite continuum CFT with Lorentz invariance.

Whilst this work was in preparation, results in higher-dimensional non-relativistic and massless relativistic free fermion models have been obtained [51], where efficient semi-classical-type techniques are developed for averages of single local observables. Here we provide instead a full quantum calculation; this allows us to go beyond averages of single observables and to obtain a rather complete description of the Klein-Gordon model. This includes a proof and analysis of the non-equilibrium density matrix, the full current fluctuation spectrum and the approach to the steady state. We supplement our analysis with new exact results for free Dirac fermions.

A key motivation for the present work is to compare and contrast the free-field limit with our previous results in the strongly interacting regime [40]. We will show that in higher dimensions, the critical Klein-Gordon model does not reproduce the strongly interacting results of [40], and nor should it: there is a fundamental distinction between the behavior of free-fields and interacting CFT in $d>1$. This is different from the one-dimensional case, where the free-field limit captures the general CFT results. A crucial distinction in higher-dimensions is that the Klein-Gordon model contains infinitely many conserved quantities, in stark contrast to a generic higher-dimensional CFT; this has a direct impact on the non-equilibrium density matrix and thus physical observables. Indeed, it is well known that harmonic crystals exhibit anomalous non-equilibrium transport properties due to this proliferation of conservation laws, including the inapplicability of Fourier's law. The recent results of [33, 34, 40] show that anomalous transport also occurs in interacting quantum critical systems due to the presence of ballistic transport. The present paper indicates that in higher dimensions, quantum critical systems display more acutely anomalous behavior: there is a disconnect between the free-field limit and the generic interacting problem. We shall discuss the role of interactions in bridging these results. Our exact free-field results may also provide useful benchmarks for numerical simulations in more than one dimension.

The specific situation we shall consider is one where the two semi-infinite halves of the model (i.e. the "leads"), at $x^{1}<0$ and $x^{1}>0$, are independently thermalized at temperatures $T_{\mathrm{L}}$ and $T_{\mathrm{R}}$, respectively [33, 40]. They are then brought into instantaneous contact along the $d-1$ dimensional hypersurface $x^{1}=0$ and are allowed to evolve unitarily; see Fig. 1. We consider the impact of different initial conditions at the connection hypersurface, including both free and fixed. We find the following results, some of which were also found (in different forms) in [51]:

- At late times, a steady state carrying an energy current emerges. It is described by thermally distributed modes of positive and negative longitudinal momenta with temperatures 


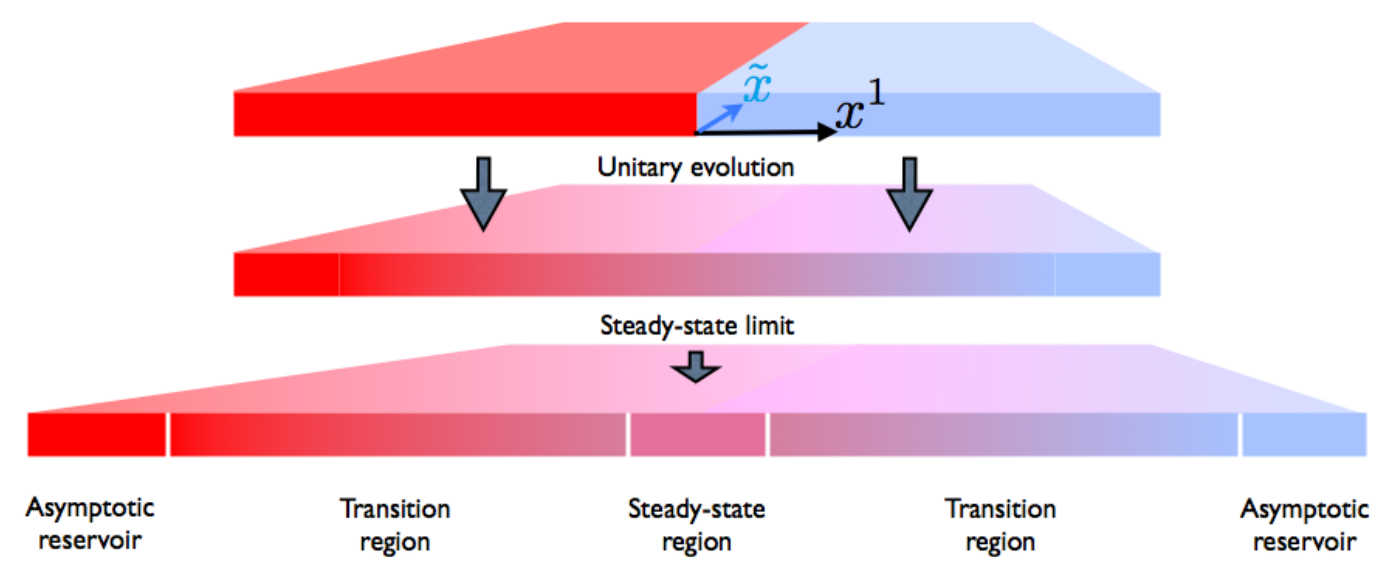

Figure 1: Two semi-infinite systems described by the massive Klein-Gordon model in $d$ dimensions are independently thermalized at temperatures $T_{\mathrm{L}}$ and $T_{\mathrm{R}}$. They are brought into instantaneous contact along the $d-1$ dimensional hypersurface $x^{1}=0$. At late times a spatially homogeneous non-equilibrium steady state (NESS) emerges carrying a non-vanishing energy current.

$T_{\mathrm{L}}$ and $T_{\mathrm{R}}$ respectively. The form of the steady state is independent of the initial conditions on the hypersurface and the exact energy current separates into a difference of a function evaluated at $T_{\mathrm{L}}$ and $T_{\mathrm{R}}$ respectively. Similar observations were made in other free-particle models in one dimension $[7,8,9,10,11,37]$ and in higher dimensions [51]; this separation is in contrast to the behavior expected for an interacting CFT in $d>1$ [40].

- One may describe the exact steady state using a density matrix. The density matrix has the form $\rho_{\mathrm{s}}=\mathrm{e}^{-\hat{W}}$ where $\hat{W}$ is a conserved quantity. This conserved quantity is not local: it is rather bilinear in the fields, with an algebraically decaying kernel. This points to asymptotically algebraic correlations for certain observables in the non-equilibrium steady state.

- All the cumulants of the total energy transferred, within a time $t$ and through a transverse hypersurface of area $A$, scale like $t A$ as $t, A \rightarrow \infty$. The scaled cumulants are obtained by dividing by $t A$ and taking the limit $t, A \rightarrow \infty$. The exact generating function for all the scaled cumulants is that of a continuum of Poisson processes with a weight function $\omega(q)$ that is analytically determined; see Eq. (23). That is, the scaled cumulants are given by $\int \mathrm{d} q \omega(q) q^{k}$, including the average energy current at $k=1$.

- The averages of generic local observables approach the NESS with a power-law in time $t$, where the exponent depends on the initial conditions at the connection hypersurface. The generic leading order response is faster, $t^{-1}$, for free initial boundary conditions, and slower, $t^{-\frac{1}{2}}$, for other initial conditions. The vanishing of the leading order contribution may give rise to a faster approach.

- The energy current and energy density approach their steady-state values faster than the generic result in the case of fixed boundary conditions: $t^{-2}$ for the average of the energy current, and $t^{-1}$ for the average of the energy density, with oscillating factors. They agree with the generic result for free boundary conditions. 
- For free initial boundary conditions, the steady state is reached immediately for any observable lying on the connection hypersurface itself and hyper-local (without derivatives) in the longitudinal direction. At the level of averages of local fields, this is similar to the results found in [51]. For fixed initial boundary conditions a similar statement holds for observables involving only single derivatives of fundamental fields in the longitudinal direction, and these statements generalize to observables involving only even or only odd derivatives of fundamental fields.

- In the massless case, fields integrated along the transverse direction take their steady state values immediately inside the light-wedge. This can be explained by a suitable dimensional reduction of the Klein-Gordon theory and results for $d=1$ CFT. A similar dimensional reduction was at the basis of the methods used in [51]; in Appendix G we provide a full operator statement of this dimensional reduction within the Klein-Gordon model.

- At space-time positions far from the connection event and within the light-wedge, a semiclassical analysis can be used, where averages receive independent contributions from waves traveling at their respective group velocities, carrying the thermal information from the left and right reservoirs. In this region, averages of local observables take simple forms, and in particular are monotonic with time. A similar semiclassical picture is used in [51].

The layout of this paper is as follows. In Section 2 we describe the NESS in arbitrary dimensions, including exact results for the average energy current, the average energy density, and the entire spectrum of fluctuations. We interpret the results in terms of a Poisson process. We also analyze the locality properties of the non-equilibrium density matrix. In Section 3 we obtain exact results for the complete time-evolution and extract the power-law approach to the NESS, including the effects of the initial boundary conditions. We also consider the time evolution of non-generic observables, including analytical results based on a suitable dimensional reduction of the Klein-Gordon model. We complement our exact calculations with semiclassical results that are valid in the limit of large distances and long times. We conclude in Section 5, presenting arguments for how the presence of weak interactions will modify the free-field results engendering a crossover to the hydrodynamic regime [40]. We include supporting calculations in Appendices A-H, and also present the free Dirac fermion results in Appendix I.

\section{Exact steady-state density matrix, averages and fluctuations}

In this section we derive the exact average energy current and energy density, as well as the full fluctuation spectrum, in the steady state that occurs as the final configuration after the two semi-infinite systems have been brought into contact. We employ the exact steady state density matrix which we initially motivate on physical grounds and derive later in Section 3.

First, let us discuss the occurrence of a non-trivial steady state, the notion of a steady state density matrix, and its expected form in free models.

As is suggested by Fig. 1, one expects that, due to causality and finite propagation speeds, there will be at all times regions which have not been affected by the quench and are still thermal. A long time after the quench, these effective reservoirs are far from the connection hypersurface. Hence, one would expect very small energy density gradients in the region around the connection hypersurface, and zero gradients at infinite time. This implies that any diffusive 
energy transport will be suppressed, so that only ballistic transport will occur in the steady state. Ballistic transport is expected to occur, for instance, when the current is a conserved density, due to Mazur's inequality [52, 53], an indicator of near-equilibrium ballistic transport. This is the case for the energy current in any relativistic quantum field theory as it is the density of the momentum, a conserved quantity. Hence, in this case we expect to have a non-trivial current carrying steady state in the partitioning approach. This argument holds in any dimension, and the emergence of a non-zero current in field theory has been explicitly shown in one-dimensional CFT [33, 34], in higher-dimensional CFT [40], and in higher-dimensional free fermion models [51]. Section 3 provides a proof in the higher-dimensional Klein-Gordon theory. Ballistic energy transport is also expected in integrable lattice models that are not necessarily in the scaling limit described by QFT [53]. This has been explicitly shown only in one-dimensional models admitting free-boson or free-fermion representations, such as the harmonic chain [41, 6, 44], free-fermionic conductors $[7,8]$ and the XY model $[9,10,11]$.

The steady state occurs, after an infinite time, in a region around the connection hypersurface that is small as compared to the size of the system; see Fig. 1. The steady state is a map

$$
\mathcal{O} \mapsto\langle\mathcal{O}\rangle_{\mathrm{s}}
$$

from observables lying in the steady state region, to their averages, calculated in the steady-state limit; see Eq. (8) below. This map does not provide any precise information about the rest of the system. The steady-state region can be taken as any volume of space extending a finite distance $\ell$ from the hypersurface (here we concentrate solely on bulk observables, with the boundaries of the system being asymptotically far from the observables), and in the steady state limit we take $\ell \ll t \ll L$, where $t$ is the time after the connection, and $L$ the linear size of the system.

In the limit $L \rightarrow \infty$ followed by $t \rightarrow \infty$, although the region is small as compared to the system, one can take $\ell$ as large as possible. In fact, in order to describe the full steady state and analyze, for instance, the large-distance behavior of correlation functions, one must take $\ell \rightarrow \infty$. The steady state then lies on an infinite (open) system.

A standard way of describing quantum averages is by using a density matrix and tracing over a Hilbert space $\mathcal{H}$ :

$$
\langle\mathcal{O}\rangle_{\mathrm{s}}=\frac{\operatorname{Tr}_{\mathcal{H}}\left(\rho_{\mathrm{s}} \mathcal{O}\right)}{\operatorname{Tr}_{\mathcal{H}}\left(\rho_{\mathrm{s}}\right)}
$$

Intuitively, one may extract two ingredients in this description: the density matrix $\rho_{\mathrm{s}}$ and the tracing operation $\operatorname{Tr}_{\mathcal{H}}$. Physically, one may expect the former to contain the information of the state the system is in, and the latter that of the dynamics of the system. Steady states of open quantum systems present problems: in non-equilibrium steady states, the volume is intrinsically infinite, and there are no a priori finite-volume underlying descriptions giving discrete sets of vectors in which to define the trace operation $\operatorname{Tr}_{\mathcal{H}}$ and the density matrix $\rho_{\mathrm{s}}$.

Nevertheless, as advocated in [12], one may use a continuum of (un-normalizable) scattering states, instead of a discrete basis. In an IR-free QFT, these are the asymptotic states, representing massive relativistic particles that are asymptotically freely propagating. In the case of energy transfer, the eigenvalues of the steady state density matrix $\rho_{\mathrm{S}}$ on the asymptotic states were proposed in $[33,36]$ in one-dimensional models. They describe weights according to the total energy of particles with positive and negative momenta. Weights for particles of positive (negative) momenta are Boltzmann weights at temperature $T_{\mathrm{L}}\left(T_{\mathrm{R}}\right)$. This description is a generalization of what was found in the free-fermionic and XY chains $[7,9,10,11]$. 
This description is, in general, not enough to fully determine the map $\mathcal{O} \mapsto\langle\mathcal{O}\rangle_{\mathrm{s}}$ associated with the density matrix as per (1): because of the continuum of asymptotic states, one needs to determine their density in order to define the trace operation $\operatorname{Tr}_{\mathcal{H}}$, carrying the dynamical information. This is in general a hard problem. A solution was proposed for 1+1-dimensional integrable massive QFT in [35] via (a generalization of) the thermodynamic Bethe ansatz, and the problem is solved thanks to chiral factorization in one-dimensional CFT [33, 34]. The answer is quite different in higher-dimensional interacting conformal field theory, where the long time behavior is not characterized by a quasiparticle description: it was found recently in [40] that the emergent steady state must be a Lorentz boosted thermal state, with the rest-frame temperature and boost velocity determined by gauge-gravity duality and relativistic hydrodynamics.

Here we consider the Klein-Gordon theory of a free boson. Due to the lack of interaction between particles, one may determine the density of asymptotic states by thermalization of the independent Fock modes. In Section 3 we explicitly show that this steady state, in the $d$-dimensional Klein-Gordon theory, is indeed that resulting from the partitioning approach, paralleling the situation in one-dimensional quadratic and free-particle models. Below we define and use this density matrix in order to obtain steady-state averages of the energy current and energy density, as well as all the long-time fluctuations of the energy transfer. We then analyze the non-local form of the density matrix.

\subsection{The exact steady-state density matrix}

In order to fix notations, we recall that the massive Klein-Gordon model describes canonical fields $\phi(x)$ and $\pi(x)$ (for $x \in \mathbb{R}^{d}$ the space coordinate) with equal-time commutation relations

$$
[\phi(x), \phi(y)]=[\pi(x), \pi(y)]=0, \quad[\phi(x), \pi(y)]=\mathrm{i} \delta^{d}(x-y)
$$

and Hamiltonian

$$
H=\frac{1}{2} \int \mathrm{d}^{d} x:\left(\pi(x)^{2}+(\nabla \phi(x))^{2}+m^{2} \phi(x)^{2}\right): .
$$

We introduce the Fourier modes $A_{p}, A_{p}^{\dagger}$ through

$$
\phi(x)=\int D p\left(A_{p} \mathrm{e}^{\mathrm{i} p \cdot x}+A_{p}^{\dagger} \mathrm{e}^{-\mathrm{i} p \cdot x}\right), \quad \pi(x)=-\mathrm{i} \int D p E_{p}\left(A_{p} \mathrm{e}^{\mathrm{i} p \cdot x}-A_{p}^{\dagger} \mathrm{e}^{-\mathrm{i} p \cdot x}\right),
$$

where $p \cdot x=\sum_{j=1}^{d} p^{j} x^{j}, E_{p}=\sqrt{p^{2}+m^{2}}$ is the relativistic energy for a particle of momentum $p$, and $D p=\frac{\mathrm{d}^{d} p}{(2 \pi)^{d} 2 E_{p}}$ is the Lorentz invariant measure. The canonical commutation relations (2) imply

$$
\left[A_{p}, A_{q}\right]=0, \quad\left[A_{p}, A_{q}^{\dagger}\right]=(2 \pi)^{d} 2 E_{p} \delta^{d}(p-q)
$$

and the Hamiltonian is diagonalized as $H=\int D p E_{p} A_{p}^{\dagger} A_{p}$. The associated Hilbert space is the Fock space over the algebra (5), with vacuum $\mid$ vac $\rangle$ defined by $A_{p} \mid$ vac $\rangle=0$.

The total Hamiltonians for the separate left and right subsystems can be expressed as

$$
H_{\mathrm{L}, \mathrm{R}}=\frac{1}{2} \int_{x_{1} \lessgtr 0} \mathrm{~d}^{d} x:\left(\pi(x)^{2}+(\nabla \phi(x))^{2}+m^{2} \phi(x)^{2}\right): .
$$

The initial density matrix, where the left and right subsystems are independently thermalized at inverse temperatures $\beta_{\mathrm{L}}$ and $\beta_{\mathrm{R}}$ respectively, is then

$$
\rho_{0}=\mathrm{e}^{-\beta_{\mathrm{L}} H_{\mathrm{L}}-\beta_{\mathrm{R}} H_{\mathrm{R}}} .
$$


In the quench setup, we instantaneously connect the two halves together and let the full system evolve unitarily with $e^{i H t}$ for a long period of time. Given a product of local observables $\mathcal{O}$, whose support is finite, its average in the steady state is defined by the following limit, if it exists:

$$
\langle\mathcal{O}\rangle_{\mathrm{s}}=\lim _{t \rightarrow \infty} \operatorname{Tr}\left(\mathfrak{n}\left[\rho_{0}\right] \mathrm{e}^{\mathrm{i} H t} \mathcal{O} \mathrm{e}^{-\mathrm{i} H t}\right),
$$

where we use $\mathfrak{n}[\rho]=\rho / \operatorname{Tr}(\rho)$. Below we also use the notation $\langle\mathcal{O}\rangle=\operatorname{Tr}\left(\mathfrak{n}\left[\rho_{0}\right] \mathcal{O}\right)$ for averages in the initial state, and operators are implicitly evolved with the dynamics generated by $H$ : $\mathcal{O}(t)=\mathrm{e}^{\mathrm{i} H t} \mathcal{O} \mathrm{e}^{-\mathrm{i} H t}$.

According to the above discussion, we expect that the limit (8) exists for any product of local observables $\mathcal{O}$, and that its result can be calculated using the steady-state density matrix

$$
\rho_{\mathrm{s}}:=\exp \left[-\beta_{\mathrm{L}} \int_{p^{1}>0} D p E_{p} A_{p}^{\dagger} A_{p}-\beta_{\mathrm{R}} \int_{p^{1}<0} D p E_{p} A_{p}^{\dagger} A_{p}\right] .
$$

This describes thermally distributed modes of positive and negative longitudinal momenta with temperatures $T_{\mathrm{L}}$ and $T_{\mathrm{R}}$ respectively. The steady-state density matrix is both stationary and homogeneous. Expectation values in the NESS are given by

$$
\langle\mathcal{O}\rangle_{\mathrm{s}}=\operatorname{Tr}\left(\mathfrak{n}\left[\rho_{\mathrm{s}}\right] \mathcal{O}\right)
$$

These may be evaluated using the mode expansion (4), Wick's theorem and the contractions

$$
\operatorname{Tr}\left(\mathfrak{n}\left[\rho_{\mathrm{s}}\right] A_{p} A_{q}^{\dagger}\right)=\frac{(2 \pi)^{d} 2 E_{p} \delta^{d}(p-q)}{1-\mathrm{e}^{-W(p)}}, \quad \operatorname{Tr}\left(\mathfrak{n}\left[\rho_{\mathrm{s}}\right] A_{p}^{\dagger} A_{q}\right)=\frac{(2 \pi)^{d} 2 E_{p} \delta^{d}(p-q)}{\mathrm{e}^{W(p)}-1} .
$$

The latter are obtained using the cyclic property of the trace, the canonical commutation relations (5), and the exchange relation $\rho_{\mathrm{s}} A_{p}=\mathrm{e}^{-W(p)} A_{p} \rho_{\mathrm{s}}$, where

$$
W(p):= \begin{cases}\beta_{\mathrm{L}} E_{p} & \left(p^{1}>0\right) \\ \beta_{\mathrm{R}} E_{p} & \left(p^{1}<0\right)\end{cases}
$$

In these notations

$$
\rho_{\mathrm{s}}=\exp \left[-\int D p W(p) A_{p}^{\dagger} A_{p}\right]
$$

In Section 3 we will explicitly show that the steady state density matrix $\rho_{\mathrm{s}}$ arises at late times in the quench problem depicted in Fig. 1. In addition, we will provide the complete time evolution of general averages of local observables. In the remainder of this section we focus on the consquences of the exact steady state density matrix, providing results for the average energy density, energy current and the complete distribution of energy current fluctuations.

\subsection{Steady-state averages of the energy current and energy density}

From the above description, the averages of the energy current density $T^{01}$ and the energy density $T^{00}$ are readily evaluated. The stress-energy tensor is given by

$$
T^{\mu \nu}=: \partial^{\mu} \phi \partial^{\nu} \phi-\frac{1}{2} \eta^{\mu \nu} \partial^{\rho} \phi \partial_{\rho} \phi:
$$


Using equations (4), (10) and (11), the result is

$$
\left\langle T^{\mu \nu}\right\rangle_{\mathrm{s}}=\int D p \frac{2 p^{\mu} p^{\nu}}{\mathrm{e}^{W(p)}-1},
$$

where $p^{0}=E_{p}$. In particular, the energy current density is that expected for a free bosonic model, averaging the momentum with bosonic filling fractions dependent on its sign:

$$
\left\langle T^{01}\right\rangle_{\mathrm{s}}=\int \frac{\mathrm{d}^{d} p}{(2 \pi)^{d}}\left|p^{1}\right|\left(\frac{\Theta\left(p^{1}\right)}{\mathrm{e}^{\beta_{\mathrm{L}} E_{p}}-1}-\frac{\Theta\left(-p^{1}\right)}{\mathrm{e}^{\beta_{\mathrm{R}} E_{p}}-1}\right) .
$$

One may evaluate the averages of the energy current and the energy density explicitly by performing the angular integrals; see Appendix A. The results may be expressed in various ways:

$$
\begin{aligned}
\left\langle T^{01}\right\rangle_{\mathrm{s}} & =\frac{\Gamma\left(\frac{d}{2}\right)}{4 \pi^{d / 2+1}(d-1) !} \int_{0}^{\infty} \mathrm{d} p p^{d} \frac{\sinh \left(\frac{\beta_{\mathrm{R}}-\beta_{\mathrm{L}}}{2} E_{p}\right)}{\sinh \left(\frac{\beta_{\mathrm{R}}}{2} E_{p}\right) \sinh \left(\frac{\beta_{\mathrm{L}}}{2} E_{p}\right)} \\
& =\frac{d \Gamma\left(\frac{d}{2}\right)}{2 \pi^{d / 2+1}}\left(\zeta_{\frac{m}{T_{\mathrm{L}}}}(d+1) T_{\mathrm{L}}^{d+1}-\zeta_{\frac{m}{T_{\mathrm{R}}}}(d+1) T_{\mathrm{R}}^{d+1}\right), \\
\left\langle T^{00}\right\rangle_{\mathrm{s}} & =\frac{\Gamma\left(\frac{d+1}{2}\right)}{4 \pi^{(d+1) / 2}(d-1) !} \int_{0}^{\infty} \mathrm{d} p p^{d-1} E_{p} \frac{\cosh \left(\frac{\beta_{\mathrm{R}}-\beta_{\mathrm{L}}}{2} E_{p}\right)}{\sinh \left(\frac{\beta_{\mathrm{R}}}{2} E_{p}\right) \sinh \left(\frac{\beta_{\mathrm{L}}}{2} E_{p}\right)} \\
& =\frac{d \Gamma\left(\frac{d+1}{2}\right)}{2 \pi^{(d+1) / 2}}\left(\tilde{\zeta}_{\frac{m}{T_{\mathrm{L}}}}(d+1) T_{\mathrm{L}}^{d+1}+\tilde{\zeta}_{\frac{m}{T_{\mathrm{R}}}}(d+1) T_{\mathrm{R}}^{d+1}\right),
\end{aligned}
$$

where we define the functions

$$
\zeta_{a}(z):=\frac{1}{\Gamma(z)} \int_{0}^{\infty} \mathrm{d} p \frac{p^{z-1}}{e^{\sqrt{p^{2}+a^{2}}}-1}, \quad \tilde{\zeta}_{a}(z):=\frac{1}{\Gamma(z)} \int_{0}^{\infty} \mathrm{d} p \frac{p^{z-2} \sqrt{p^{2}+a^{2}}}{e^{\sqrt{p^{2}+a^{2}}}-1} .
$$

The averages in Eq. (15) separate into sums and differences of functions of $T_{\mathrm{L}}$ and $T_{\mathrm{R}}$, as is the case in $1+1$ CFT [33, 34] and free particle models $[7,9,10,11,51]$. This is a consequence of the triviality of the scattering matrix and differs from the generic case of higher-dimensional CFT [40]. In the massless limit (corresponding to $a=0$ ) both functions in Eq. (16) specialize to the Riemann zeta function, $\zeta_{0}(z)=\tilde{\zeta}_{0}(z)=\zeta(z)$. In particular, setting $d=1$ and $m=0$, and using $\zeta(2)=\pi^{2} / 6$ with $\Gamma(1 / 2)=\sqrt{\pi}$, we find that the coefficients of the powers of temperature all specialize to $\pi / 12$ (both for $\left\langle T^{00}\right\rangle_{\mathrm{s}}$ and $\left\langle T^{01}\right\rangle_{\mathrm{s}}$ ) as required for a $1+1$ CFT with central charge $c=1[33,34]$. The $T^{d+1}$ temperature dependence appearing in Eq. (15) when $m=0$ is analagous to the Stefan-Boltzmann law for black body radiation [40]. More generally, in the massive case this temperature dependence is multiplied by a function of $m / T$. Setting $T_{\mathrm{L}}=T$ and $T_{\mathrm{R}}=0$ one may denote $\left\langle T^{01}\right\rangle_{\mathrm{s}}=\left\langle T^{01}\right\rangle_{\mathrm{s}}^{m=0} f_{d}^{01}(m / T)$ and $\left\langle T^{00}\right\rangle_{\mathrm{s}}=\left\langle T^{00}\right\rangle_{\mathrm{s}}^{m=0} f_{d}^{00}(m / T)$. The functions $f_{d}^{01}(m / T)$ and $f_{d}^{00}(m / T)$ are plotted in Fig. 2 for different spatial dimensions.

Results for the free massive Dirac model are presented in Appendix I.

\subsection{Exact cumulant generating function of the energy transfer}

The observables of most interest in the general theory of non-equilibrium steady states are the scaled cumulants of the quantity being transferred. The generating function of these cumulants is 

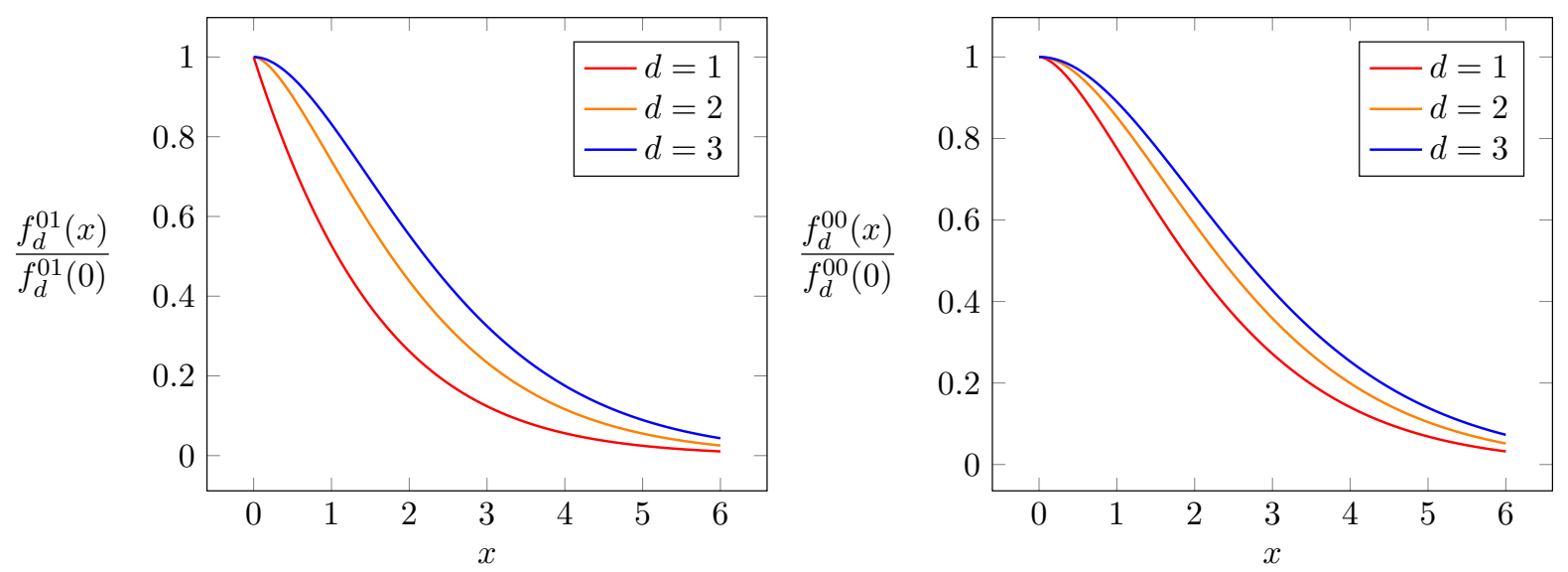

Figure 2: Left: Energy current function, $f_{d}^{01}(x)(x=m / T)$ for $d=1,2,3$. Right: Energy density function, $f_{d}^{00}(x)$ for $d=1,2,3$. Both are exponentially decaying at large mass $m$ (low temperature $T$ ).

related to the large-deviation function [3], which is thought to be a good candidate for replacing the free energy as a "thermodynamic potential" in non-equilibrium systems. The cumulants provide the full long-time statistics of the transferred quantity, and hence much more information about the physics of the transfer process.

The fundamental definition of the scaled cumulants (here in the case of energy transfer) is as follows. Suppose we measure the energy $q$ transferred from the left to the right a time $t$ after the connection. In order to have finite energy transfer in finite time, we assume that the $d-1$ dimensional transverse directions (transverse to the flow) have linear size $L$. The quantity $q$ is a random quantity, and we may represent the associated probability measure by $\Omega_{t, L}$. According to standard statistical definitions, the cumulants $\left\langle q^{n}\right\rangle_{\Omega_{t, L}}^{\text {cumul }}$ of the random variable $q$ may be defined from its averages by the generating function $\sum_{n=1}^{\infty} \frac{z^{n}}{n !}\left\langle q^{n}\right\rangle_{\Omega_{t, L}}^{\text {cumul }}=\log \left\langle\mathrm{e}^{z q}\right\rangle_{\Omega_{t, L}}$. The scaled cumulants, and scaled cumulant generating function (SCGF), are then defined as, respectively,

$$
c_{n}:=\lim _{\substack{t \rightarrow \infty \\ L \rightarrow \infty}} \frac{1}{t L^{d-1}}\left\langle q^{n}\right\rangle_{\Omega_{t, L}}^{\text {cumul }}, \quad F(z)=\sum_{n=1}^{\infty} \frac{z^{n}}{n !} c_{n} .
$$

This assumes that the cumulants of the variable $q$ scale proportionally to the time and to the transverse area at large time and large transverse area.

In order to have a working definition of $c_{n}$, we would need to define more precisely $\Omega_{t, L}$. The exact definition of the measure $\Omega_{t, L}$ in quantum systems is subtle because, contrary to the case of classical systems, it requires a precise description of the quantum measuring process and of its influence on the system. Here we will not discuss these subtleties - for discussions see for instance $[30,34]$.

Instead, we will adopt a simple and intuitive expression for $c_{n}$, which is frequently used in the literature and is expected to arise as a result of various measurement protocols. This expression gives the scaled cumulants in terms of the connected correlation functions of the integrated energy current density. That is, cumulants are expressed as connected correlation functions of 
the current density $T^{01}(x, t)=T^{01}\left(x^{1}, \tilde{x}, t\right)$ integrated over the coordinates $\tilde{x}$ parametrizing the transverse direction and over time $t$, evaluated on the connection hypersurface $x^{1}=0$ :

$$
c_{n}=\lim _{\epsilon \rightarrow 0^{+}} \int \prod_{j=1}^{n-1} \mathrm{~d}^{d-1} \tilde{x}_{j} \int_{-\infty}^{\infty} \prod_{j=1}^{n-1} \mathrm{~d} t_{j}\left\langle T^{01}\left(0, \tilde{x}_{n-1}, t_{n-1}^{(\epsilon)}\right) \cdots T^{01}\left(0, \tilde{x}_{1}, t_{1}^{(\epsilon)}\right) T^{01}(0,0,0)\right\rangle_{\mathrm{s}}^{\text {conn. }} .
$$

where $t_{j}^{(\epsilon)}=t_{j}+i j \epsilon$. This expression should be interpreted as the scaled average of the $n^{\text {th }}$ power of the total energy passing through the hypersurface in a long time and for a large transverse area. One regularizes the UV singularities arising from colliding fields by imposing an imaginary time ordering to the factors: hence the $\epsilon$-regularization in (18). The result is finite and real.

The integrals in Eq. (18) are in general hard to evaluate. One can however evaluate the full SCGF directly by using the extended fluctuation relations (EFR), derived in [61] and shown there to hold in free models of any dimensionality. The EFR state that

$$
F(z)=\int_{0}^{z} \mathrm{~d} y J_{\mathrm{E}}\left(\beta_{\mathrm{L}}-y, \beta_{\mathrm{R}}+y\right)
$$

where $J_{\mathrm{E}}\left(\beta_{\mathrm{L}}, \beta_{\mathrm{R}}\right)=\left\langle T^{01}\right\rangle_{\mathrm{s}}$ is the steady-state energy current density as a function of the inverse temperatures. In particular, the cumulants can be evaluated in terms of derivatives of the current, thus providing expressions for the non-trivial multiple integrals (18) directly from the exact expression (15). Using the mode expansion (4) in the stress-energy tensor (12), along with Wick's theorem and the contractions (11), we have evaluated explicitly $c_{2}$ using (18), and verified that it is in agreement with the coefficient of $z^{2} / 2$ on the right-hand side of (19).

A convenient way of representing the SCGF that has a clear physical meaning as the transport of energy quanta is via a sum of independent Poisson processes. The SCGF for a single Poisson process representing energy transfers by quanta $q \in \mathbb{R}$ is $\mathrm{e}^{z q}-1$, and the SCGF is in general additive for independent processes. Hence, we wish to express $F(z)$ as

$$
F(z)=\int \mathrm{d} q \omega(q)\left(\mathrm{e}^{z q}-1\right)
$$

In order for this to have an interpretation as a sum of independent Poisson processes, the weight $\omega(q)$ must be positive. If this is the case, then (20) implies that the long-time scaled energy transfer can be fully reproduced by a classical process whereby quanta of energy between $|q|$ and $|q|+d q$, traveling towards the right $(q>0)$ or the left $(q<0)$ in a cross section $\mathrm{d}^{d-1} \tilde{x}$, are distributed uniformly so that they cross the measuring hypersurface with a flat probability weighted by $\mathrm{d}^{d-1} \tilde{x} \mathrm{~d} q \omega(q)$.

Following [35] we can express the weight as a Fourier transform of the current,

$$
\omega(q)=\frac{1}{q} \int \frac{\mathrm{d} \lambda}{2 \pi} J_{\mathrm{E}}\left(\beta_{\mathrm{L}}-i \lambda, \beta_{\mathrm{R}}+i \lambda\right) \mathrm{e}^{-\mathrm{i} \lambda q} .
$$

Using (14) in the form

$$
J_{\mathrm{E}}\left(\beta_{\mathrm{L}}, \beta_{\mathrm{R}}\right)=\int \frac{\mathrm{d}^{d} p}{(2 \pi)^{d}}\left|p^{1}\right| \sum_{n=1}^{\infty}\left(\Theta\left(p^{1}\right) \mathrm{e}^{-n \beta_{\mathrm{L}} E_{p}}-\Theta\left(-p^{1}\right) \mathrm{e}^{-n \beta_{\mathrm{R}} E_{p}}\right)
$$


and performing explicitly the $\lambda$ and $p$ integrals, one obtains

$$
\begin{aligned}
\omega(q) & =\sum_{n=1}^{\infty} \int \frac{\mathrm{d} p^{2} \cdots \mathrm{d} p^{d}}{(2 \pi)^{d} n^{2}}\left(\mathrm{e}^{-\beta_{\mathrm{L}} q} \Theta\left(q-n \sqrt{\tilde{p}^{2}+m^{2}}\right)+\mathrm{e}^{\beta_{\mathrm{R}} q} \Theta\left(-q-n \sqrt{\tilde{p}^{2}+m^{2}}\right)\right) \\
& =\left(2^{d} \pi^{\frac{d+1}{2}} \Gamma\left(\frac{d+1}{2}\right)\right)^{-1} \sum_{n=1}^{[|q| / m]} \frac{1}{n^{d+1}}\left(q^{2}-n^{2} m^{2}\right)^{\frac{d-1}{2}} \cdot \begin{cases}\mathrm{e}^{-\beta_{\mathrm{L}} q} & (q>0) \\
\mathrm{e}^{\beta_{\mathrm{R}} q} & (q<0)\end{cases}
\end{aligned}
$$

where $\tilde{p}^{2}=\sum_{i=2}^{d}\left(p^{i}\right)^{2}$ and $[|q| / m]$ is the integer part of $|q| / m$. This is clearly positive, hence the long-time fluctuations are correctly represented by a family of independent Poisson processes. For $d=1$ the expression reduces to that obtained for the one-dimensional Ising model [37] except for the absence of the fermionic sign factor $(-1)^{n-1}$.

The SCGF can be evaluated using (20) as an infinite sum of modified Bessel functions:

$$
F(z)=f\left(z, \beta_{\mathrm{L}}\right)+f\left(-z, \beta_{\mathrm{R}}\right),
$$

where

$$
f(z, \beta)=\left(\frac{m}{2}\right)^{\frac{d}{2}} \sum_{n=1}^{\infty} \frac{1}{(\pi n)^{1+\frac{d}{2}}}\left((\beta-z)^{-\frac{d}{2}} K_{d / 2}(n m(\beta-z))-\beta^{-\frac{d}{2}} K_{d / 2}(n m \beta)\right) .
$$

Note that the symmetry $\beta_{\mathrm{L}} \mapsto \beta_{\mathrm{R}}+z, \beta_{\mathrm{R}} \mapsto \beta_{\mathrm{L}}-z$ is a consequence of the EFR (19).

In the massless limit (23) yields

$$
\omega(q) \stackrel{m=0}{=} \frac{\zeta(d+1)}{2^{d} \pi^{\frac{d+1}{2}} \Gamma\left(\frac{d+1}{2}\right)} q^{d-1} \cdot \begin{cases}\mathrm{e}^{-\beta_{\mathrm{L}} q} & (q>0) \\ \mathrm{e}^{\beta_{\mathrm{R}} q} & (q<0) .\end{cases}
$$

For $d=1$ this reproduces the $1+1$ CFT result [33, 34] for central charge $c=1$. We see that the energy dependence of the weights for the independent energy quanta are determined not only by the Boltzman distribution, as in the $d=1$ case, but also by a factor $q^{d-1}$ due to the impact of the extended transverse area. Using (26) the massless limit of the SCGF can be directly evaluated:

$$
F(z) \stackrel{m=0}{=} \frac{\Gamma\left(\frac{d}{2}\right) \zeta(d+1)}{2 \pi^{1+\frac{d}{2}}}\left(\left(\beta_{\mathrm{L}}-z\right)^{-d}+\left(\beta_{\mathrm{R}}+z\right)^{-d}-\beta_{\mathrm{L}}^{-d}-\beta_{\mathrm{R}}^{-d}\right) .
$$

Explicitly, the cumulants in the massless limit are

$$
c_{n}=\frac{\Gamma(d+n) \zeta(d+1)}{2^{d} \pi^{\frac{d+1}{2}} \Gamma\left(\frac{d+1}{2}\right)}\left(\beta_{\mathrm{L}}^{-d-n}+(-1)^{n} \beta_{\mathrm{R}}^{-d-n}\right) .
$$

Again, for $d=1$ this agrees with the known $1+1$ CFT result $[33,34]$. Results for the SCGF in the free massive Dirac models are presented in Appendix I.

\subsection{Non-locality of the steady-state density matrix and power-law correla- tions}

The steady-state density matrix is described in Eq. (9) in terms of creation and annihilation operators. It has the form $\rho_{\mathrm{s}}=\exp [-\widehat{W}]$, for a specified operator $\widehat{W}$. Naturally, $\widehat{W}$ is conserved 
by the full dynamics, $[H, \widehat{W}]=0$, as the density matrix represents a state that is stationary. It is natural to ask whether $\widehat{W}$ may be written as one of the infinitely-many local conserved charges of the Klein-Gordon theory. The locality of $\widehat{W}$, or lack thereof, is important, as it has implications for the large-distance decay of correlation functions in the steady state.

We may evaluate $\widehat{W}$ explicitly in terms of the local fields $\phi(x)$ and $\pi(x)$ by using (9) and the inversion of (4). The calculation is shown in Appendix B. The result may be expressed in the following form:

$$
\widehat{W}=\frac{\beta_{\mathrm{L}}+\beta_{\mathrm{R}}}{2} H+\frac{\beta_{\mathrm{L}}-\beta_{\mathrm{R}}}{2}\left(P_{1}+\widehat{Q}\right),
$$

where $P_{1}=\int d^{d} x \phi(x) \partial_{1} \pi(x)$ is the momentum operator in the longitudinal direction and

$$
\widehat{Q}=\int \mathrm{d}^{d} x \mathrm{~d}^{d} y: \phi(x) \pi(y): Q(x-y) .
$$

The kernel $Q(x-y)$ is given by

$$
Q(x)=-\frac{\operatorname{sign}\left(x^{1}\right)}{\pi} \int \frac{\mathrm{d}^{d-1} \tilde{p}}{(2 \pi)^{d-1}} \mathrm{e}^{\mathrm{i} \tilde{p} \cdot \tilde{x}} \int_{0}^{E_{\tilde{p}}} \mathrm{~d} \ell \mathcal{E}_{\ell, \tilde{p}} \mathrm{e}^{-\left|x^{1}\right| \ell},
$$

where $\mathcal{E}_{\ell, \tilde{p}}=\sqrt{|\tilde{p}|^{2}+m^{2}-\ell^{2}}, E_{\tilde{p}}=\mathcal{E}_{0, \tilde{p}}$, and the tilde-variables are transverse coordinates.

In (29) we recognize the terms $\frac{\beta_{\mathrm{L}}+\beta_{\mathrm{R}}}{2} H+\frac{\beta_{\mathrm{L}}-\beta_{\mathrm{R}}}{2} P_{1}$ as representing a Lorentz boost of the Hamiltonian in the longitudinal direction. These terms alone would give rise to a density matrix of a boosted thermal state with boost velocity $\frac{T_{\mathrm{L}}-T_{\mathrm{R}}}{T_{\mathrm{L}}+T_{\mathrm{R}}}$ and rest-frame temperature $\sqrt{T_{\mathrm{L}} T_{\mathrm{R}}}$. Remarkably, this has the same structure as the exact non-equilibrium steady-state density matrix of a one-dimensional CFT [33, 40], although $H$ and $P_{1}$ pertain to the higher-dimensional massive system. The third term, involving $\widehat{Q}$, is a correction to this, which further accounts for the higher dimensionality and the non-zero mass. One can check that it indeed vanishes if and only if $d=1$ and $m=0$. The third term is not an integration over a local density, and in fact, the kernel connects local fields in the bilinear expression over long distances as it does not decay exponentially. For instance, in the case with $d=1$ and $m \neq 0$, the kernel has the following large- $\left|x^{1}\right|$ asymptotic expansion:

$$
Q(x)=-\frac{m}{\pi x^{1}}(1+O(1))
$$

For $d>1$, the decay of $Q(x)$ is also $O\left(1 / x^{1}\right)$ at large $\left|x^{1}\right|$, with a coefficient that involves both the square-root of the transverse Laplacian $\sqrt{-\nabla_{\tilde{x}}^{2}} \delta^{(d-1)}(\tilde{x})$, and a regular function of the transverse coordinates $\tilde{x}$.

The algebraic decay of $Q(x)$ at large $\left|x^{1}\right|$ is the signature of large-distance algebraic correlations. These are indeed known to exist in non-equilibrium steady states [54, 55], and are usually attributed to the lack of detailed balance. The algebraic decay can be seen explicitly in the following correlation function in the one-dimensional case:

$$
\langle\phi(x) \pi(0)\rangle_{\mathrm{s}} \sim \frac{1}{4 \pi\left|x^{1}\right|} \frac{\sinh \frac{\left(\beta_{\mathrm{L}}-\beta_{\mathrm{R}}\right) m}{2}}{\sinh \frac{\beta_{\mathrm{L}} m}{2} \sinh \frac{\beta_{\mathrm{R}} m}{2}} \quad \text { as }\left|x^{1}\right| \rightarrow \infty \quad(d=1) .
$$

Interestingly, however, the correlation function $\langle\phi(x) \phi(0)\rangle_{\mathrm{s}}$ decays exponentially at large distances, emphasizing the fact that the presence of the algebraic decay depends on the observables involved. For further details of these calculations see Appendix F. 
Some comments are in order. First, we note that, at least in one-dimensional systems, it has been known for some time that the density matrix for non-equilibrium quantum steady states may be the exponential of a non-local operator. For instance, in the context of the nonequilibrium Kondo impurity model, Hershfield's density matrix [16] was analyzed in [18] and argued there to have a non-local form. Non-locality was shown explicitly in [56] for the resonantlevel impurity model. Recently, certain quantum steady states in integrable spin chains were also shown to give rise to density matrices of a non-local form [57].

Second, it is important to realize that non-equilibrium quantum steady states are not always described by density matrices of a non-local form, and do not always present algebraic correlations. Indeed, as a consequence of the results of [33] in one-dimensional CFT, and of [40] in interacting higher-dimensional CFT, the density matrix is the exponential of a local conserved charge, and correlations are exponentially decreasing in these cases. An exponential decay (at least close to equilibrium) was also proven mathematically for spin-spin correlation functions in the XY chain [58], and derived physically in the one-dimensional Ising field theory [59], despite the fact that the density matrix has a non-local form in terms of the underlying fermions.

Finally, we remark that an interesting phenomenon occurs in one-dimension: by the above discussion, we see that some correlations decay algebraically in the steady state of the massive $d=1$ Klein-Gordon model, but exponentially in the massless model.

\section{Exact time evolution and the steady state density matrix}

We now wish to evaluate explicitly the limit (8) for local operators $\mathcal{O}$, and show the form (10) of the steady state with the density matrix (9). We will use techniques based on equations of motion developed in the context of free fermionic quantum impurity problems in [30].

\section{$3.1 \quad A$ - and $B$-representations}

In the previous section, we have written the representation (4), (5) (which we will refer to as the $A$-representation) of the canonical commutation relations (2). This representation diagonalizes the Hamiltonian $H$ on the line $x^{1} \in[-\infty, \infty]$, hence it is efficient in order to evaluate timeevolved fields $\phi(x, t)=\mathrm{e}^{\mathrm{i} H t} \phi(x) \mathrm{e}^{-\mathrm{i} H t}$ and $\pi(x, t)=\mathrm{e}^{\mathrm{i} H t} \pi(x) \mathrm{e}^{-\mathrm{i} H t}$ :

$$
\begin{aligned}
\phi(x, t) & =\int D p\left(A_{p} \mathrm{e}^{-\mathrm{i} E_{p} t+\mathrm{i} p \cdot x}+A_{p}^{\dagger} \mathrm{e}^{\mathrm{i} E_{p} t-\mathrm{i} p \cdot x}\right) \\
\pi(x, t) & =-\mathrm{i} \int D p E_{p}\left(A_{p} \mathrm{e}^{-\mathrm{i} E_{p} t+\mathrm{i} p \cdot x}-A_{p}^{\dagger} \mathrm{e}^{\mathrm{i} E_{p} t-\mathrm{i} p \cdot x}\right) .
\end{aligned}
$$

However, in this representation, the Hamiltonians $H_{\mathrm{L}}$ and $H_{\mathrm{R}}$ take a complicated (although still bilinear) form. Hence, evaluating the limit (8) is a difficult task.

There is another representation (the $B$-representation) of the commutation relations (2), which diagonalizes both $H_{\mathrm{L}}$ and $H_{\mathrm{R}}$. This is the representation used for describing two boundary Klein-Gordon models, one on the negative half-line and the other on the positive half-line, respectively. In the following, we will take free boundary conditions at $x^{1}=0$, with the condition $\left(\partial_{1} \phi\right)(0)=0$, and mention how the results are modified for other boundary conditions. The 
mode expansion for the Klein-Gordon model with free boundary conditions at $x^{1}=0$ is given by

$$
\begin{aligned}
& \phi(x)=\int D p\left(B_{p} \mathrm{e}^{\mathrm{i} \tilde{p} \cdot \tilde{x}}+B_{p}^{\dagger} \mathrm{e}^{-\mathrm{i} \tilde{p} \cdot \tilde{x}}\right) 2 \cos \left(p^{1} x^{1}\right) \Theta\left(-p^{1} x^{1}\right) \\
& \pi(x)=-\mathrm{i} \int D p E_{p}\left(B_{p} \mathrm{e}^{\mathrm{i} \tilde{p} \cdot \tilde{x}}-B_{p}^{\dagger} \mathrm{e}^{-\mathrm{i} \tilde{p} \cdot \tilde{x}}\right) 2 \cos \left(p^{1} x^{1}\right) \Theta\left(-p^{1} x^{1}\right)
\end{aligned}
$$

Here, $\tilde{p} \cdot \tilde{x}=\sum_{i=2}^{d} p^{i} x^{i}$ is the dot product in the transverse direction. In this representation, the operators $B_{p}$ and $B_{p}^{\dagger}$ satisfy the same canonical commutation relations as do $A_{p}$ and $A_{p}^{\dagger}(5)$, and we have

$$
H_{\mathrm{L}, \mathrm{R}}=\int_{p^{1} \gtrless 0} D p E_{p} B_{p}^{\dagger} B_{p} .
$$

This representation is efficient in order to evaluate averages under $\rho_{0}$. We have in particular

$$
\operatorname{Tr}\left(\mathfrak{n}\left[\rho_{0}\right] B_{p} B_{q}^{\dagger}\right)=\frac{(2 \pi)^{d} 2 E_{p} \delta^{d}(p-q)}{1-\mathrm{e}^{-W(p)}}, \quad \operatorname{Tr}\left(\mathfrak{n}\left[\rho_{0}\right] B_{p}^{\dagger} B_{q}\right)=\frac{(2 \pi)^{d} 2 E_{p} \delta^{d}(p-q)}{\mathrm{e}^{W(p)}-1} .
$$

Note the similarity with (11). However, in this representation, it is much more complicated to evaluate the time-evolved operators $\mathrm{e}^{\mathrm{i} H t} \phi(x) \mathrm{e}^{-\mathrm{i} H t}$ and $\mathrm{e}^{\mathrm{i} H t} \pi(x) \mathrm{e}^{-\mathrm{i} H t}$.

In order to show convergence to the steady state, we will establish that

$$
\lim _{t \rightarrow \infty} \operatorname{Tr}\left(\mathfrak{n}\left[\rho_{0}\right] \mathrm{e}^{\mathrm{i} H t} \mathcal{O} \mathrm{e}^{-\mathrm{i} H t}\right)=\operatorname{Tr}\left(\mathfrak{n}\left[\rho_{0}\right] S(\mathcal{O})\right),
$$

for any product of operators $\mathcal{O}=\prod_{i} \mathcal{O}_{i}$, where $\mathcal{O}_{i}$ are formed by normal-ordered products of $\phi\left(x_{i}\right), \pi\left(x_{i}\right)$ and their derivatives. Here $S$ is the scattering isomorphism defined by

$$
S\left(A_{p}\right)=B_{p}, \quad S\left(A_{p}^{\dagger}\right)=B_{p}^{\dagger}, \quad S\left(\prod_{p} A_{p}^{\eta_{p}}\right)=\prod_{p} S\left(A_{p}^{\eta_{p}}\right) .
$$

Along with (37), (11) and Wick's theorem, this indeed shows (10) with (9).

\subsection{Time-evolved operators: main results}

Following the techniques used in [30], which may be used for any free model, we evaluate the time evolution with $H$ by explicitly solving the equations of motion

$$
\dot{\phi}=\pi, \quad \dot{\pi}=\left(\nabla^{2}-m^{2}\right) \phi
$$

in terms of the initial conditions on $\phi(x)$ and $\pi(x)$, and then we replace the initial conditions by their $B$-representation (35). Having time-evolved fields in the $B$-representation, we may then readily evaluate (8) using (37). The resulting integration is rather technical and we relegate it to Appendix C. We find

$$
\phi(x, t)= \begin{cases}\mathrm{e}^{\mathrm{i} H_{0} t} \phi(x) \mathrm{e}^{-\mathrm{i} H_{0} t} & \left(\left|x^{1}\right|>t\right) \\ S(\phi(x, t))+\Psi(x, t) & \left(\left|x^{1}\right|<t\right)\end{cases}
$$

where

$$
\Psi(x, t):=\left(\int D p \mathrm{e}^{\mathrm{i} \tilde{p} \cdot \tilde{x}} B_{p} G\left(x^{1}, t ; p\right)+h . c\right)
$$


$H_{0}$ is given by (75) and $S$ is the map defined by (39). The map $S$ corresponds to the steady state, and as we will see, the correction $\Psi(x, t)$ describes the approach to the steady state.

The function $G\left(x^{1}, t ; p\right)$ is the solution of a one-dimensional Klein-Gordon equation with mass-squared $|\tilde{p}|^{2}+m^{2}$, which satisfies the opposite of the initial, pre-quench boundary conditions, so that free is exchanged with fixed. An integral representation for initial free boundary conditions is

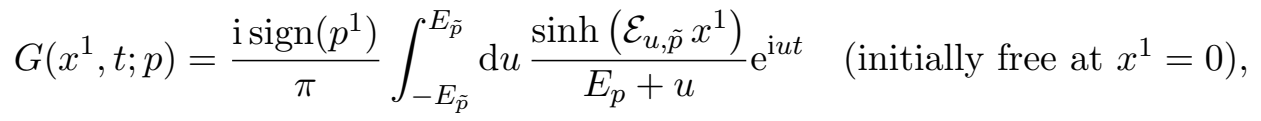

and for initial fixed boundary condition is

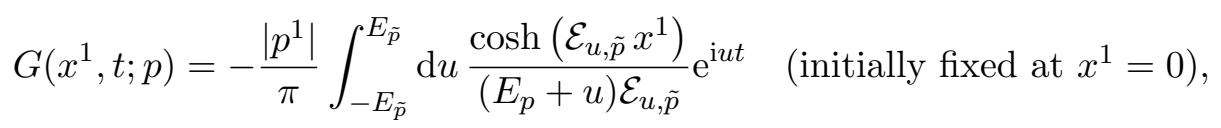

where $\mathcal{E}_{u, \tilde{p}}=\sqrt{|\tilde{p}|^{2}+m^{2}-u^{2}}$ and $E_{\tilde{p}}=\mathcal{E}_{0, \tilde{p}}$.

Let us highlight the results with the following observations:

1. According to (41), the time evolution with $H$ is exactly the same as that with $H_{0}$ whenever $\left|x^{1}\right|>t$. This is causality: beyond the light-wedge emanating from the space-time region $t=0, x^{1}=0$, it is not possible to distinguish between the dynamics generated by $H$ and that generated by $H_{0}$, as the information of the quench at $t=0, x^{1}=0$ is out of reach.

2. The correction (43) is exactly zero at $x^{1}=0$. This holds as an operator statement and subsists under the normal-ordering operation. This implies that if $\mathcal{O}$ is a local operator at $x^{1}=0$, ultra-local in the longitudinal direction (not involving $x^{1}$ derivatives), then

$$
\operatorname{Tr}\left(\mathfrak{n}\left[\rho_{0}\right] \mathcal{O}(t)\right)=\langle\mathcal{O}\rangle_{\mathrm{s}}
$$

for every $t>0$, with initially free conditions at $x^{1}=0$. That is, the steady state is reached instantaneously for such operators at $x^{1}=0$. This surprising fact is strongly connected to the choice of free boundary conditions before the quench. The interpretation is that with free boundary conditions, the fields at $x^{1}=0$ may freely reach their steady state limit, and they do so immediately.

With initially fixed boundary conditions, a similar phenomenon occurs but for local operators at $x^{1}=0$ involving only fundamental fields with single $x^{1}$ derivatives. Indeed for such operators the correction (44) vanishes. Fixed boundary conditions on $\phi$ correspond to free boundary conditions on $\partial_{1} \phi$, so that a similar interpretation holds. These observations explain why the behaviour of $G\left(x^{1}, t ; p\right)$ at $x^{1}=0$ is the opposite of the initial, pre-quench boundary condition. We note that in general, operators involving only even-derivative fundamental fields reach the steady state instantaneously on the connection hypersurface for initially free boundary conditions, and operators involving only odd derivatives do so for initially fixed boundary conditions.

3. Let $m=0$ and consider the Klein-Gordon field integrated over the perpendicular direction, $\int \mathrm{d} x^{2} \cdots \mathrm{d} x^{d} \phi(x, t)$. In this case, $\tilde{p}=0$. Since for $m=0$ we have $E_{\tilde{p}=0}=0$, then both corrections (43) and (44) vanish. That is, the massless Klein-Gordon field integrated over the perpendicular region instantaneously reaches its steady state form. Appropriately normalized, correlation functions of products of such integrated fields are finite, and 
immediately reach their steady-state value as soon as they all lie in the light-wedge. In fact, this is a special case of the general statement according to which such correlation functions are exactly described by an effective one-dimensional theory; see Appendix G. Here it is the $d=1$ free massless boson, for which the $1+1$ CFT results can be used: an instantaneous steady state everywhere within the light-cone [33, 34].

Additionally, we see that if both $m=0$ and $d=1$, then $E_{\tilde{p}=0}=0$ and the corrections (43) and (44) vanish. In this case, we again recover the 1+1-dimensional CFT result, according to which the steady state is instantaneously reached in the light-cone, for any boundary conditions.

The result (41) allows us to evaluate any time-evolved average in the local quench problem. For instance, the expression for the average energy current is reported in Appendix D in Eqs. (80) and (81).

\subsection{Long-time limit and approach to the steady state}

Consider an operator $\mathcal{O}=\prod_{i} \mathcal{O}_{i}\left(t_{i}, x_{i}\right)$ which is the product of local observables at different space-time points. Each observable $\mathcal{O}_{i}\left(t_{i}, x_{i}\right)$ is a normal-ordered product of the field $\phi\left(t_{i}, x_{i}\right)$ and/or their space-time derivatives. If the correction $\Psi(x, t)=\int D p \mathrm{e}^{\mathrm{i} \tilde{p} \cdot \tilde{x}} B_{p} G\left(x^{1}, t ; p\right)+h . c$ vanishes in the limit $t \rightarrow \infty$, then we have, from (41),

$$
\begin{aligned}
\lim _{t \rightarrow \infty} \operatorname{Tr}\left(\mathfrak{n}\left[\rho_{0}\right] \mathrm{e}^{\mathrm{i} H t} \mathcal{O} \mathrm{e}^{-\mathrm{i} H t}\right) & =\operatorname{Tr}\left(\mathfrak{n}\left[\rho_{0}\right] S\left(\mathrm{e}^{\mathrm{i} H t} \mathcal{O} \mathrm{e}^{-\mathrm{i} H t}\right)\right) \\
& =\operatorname{Tr}\left(\mathfrak{n}\left[\rho_{0}\right] \mathrm{e}^{\mathrm{i} H_{0} t} S(\mathcal{O}) \mathrm{e}^{-\mathrm{i} H_{0} t}\right) \\
& =\operatorname{Tr}\left(\mathfrak{n}\left[\rho_{0}\right] S(\mathcal{O})\right) .
\end{aligned}
$$

That is, we have recovered the relation (38), from which follows (10) with (9).

We now show that the correction $\Psi(x, t)$ provides asymptotically vanishing corrections at large $t$ in the average $\operatorname{Tr}\left(\mathfrak{n}\left[\rho_{0}\right] \mathrm{e}^{\mathrm{i} H t} \mathcal{O} \mathrm{e}^{-\mathrm{i} H t}\right)$. We concentrate first on the case where local observables $\mathcal{O}_{i}\left(t_{i}, x_{i}\right)$ are normal-orderings of powers of $\phi\left(t_{i}, x_{i}\right)$ without any derivatives. In order to evaluate the leading asymptotic correction, we again use (41) (second line) and (37) along with Wick's theorem. Note that in (41), the operator $\phi\left(t_{i}+t, x_{i}\right)$ is a sum of two contributions: its steady-state form $S\left(\phi\left(t_{i}+t, x_{i}\right)\right)$, and the correction $\Psi\left(t_{i}+t, x_{i}\right)$. In applying Wick's theorem, we have to sum over products of Wick contractions $\left(\phi\left(t_{i}+t, x_{i}\right), \phi\left(t_{j}+t, x_{j}\right)\right)$ (with possibly $i=j$ ). In order to evaluate the leading order result at large $t$, we consider terms where all Wick contractions are between steady-state forms $S\left(\phi\left(t_{i}+t, x_{i}\right)\right)$, except for one contraction involving a single correction $\Psi\left(t_{j}+t, x_{j}\right)$. Integrating over momenta and over $u$, there are oscillatory factors $\mathrm{e}^{ \pm \mathrm{i} E_{p} t}$ coming from the steady-state form, and $\mathrm{e}^{\mathrm{i} u t}$ coming from the $G$-correction. By the method of stationary phase, the leading large- $t$ result is obtained by integrating around the region where the oscillation frequency vanishes. Since $0<E_{\tilde{p}} \leq E_{p}$ for every $p$, this happens only around $p^{1}=0$ (where $E_{\tilde{p}}=E_{p}$ ) and $u= \pm E_{\tilde{p}}$.

Consider the contraction between a positive-energy term in $S\left(\phi\left(t_{i}+t, x_{i}\right)\right)$ and a negativeenergy term in $\Psi\left(t_{j}+t, x_{j}\right)$. This is of the form, after the change of variable $u \mapsto-u$,

$$
\int D p f(p) \mathrm{e}^{\mathrm{i} E_{p} t_{i}-\mathrm{i} p \cdot x_{i}+\mathrm{i} \tilde{p} \cdot \tilde{x}_{j}} \int_{-E_{\tilde{p}}}^{E_{\tilde{p}}} \mathrm{~d} u \frac{\sinh \left(\mathcal{E}_{u, \tilde{p}} x_{j}^{1}\right)}{E_{p}-u} \mathrm{e}^{\mathrm{i}\left(E_{p}-u\right) t} \mathrm{e}^{-\mathrm{i} u t_{j}}
$$


where $f(p)$ is regular and non-zero at $p^{1} \rightarrow 0$. We change variables to $s, v$ with $s=\left(p^{1}\right)^{2} /\left(2 E_{\tilde{p}}\right)$ and $v=E_{\tilde{p}}-u$ and write

$$
\int \mathrm{d}^{d} p \int^{E_{\tilde{p}}} \mathrm{~d} u \propto \int \mathrm{d}^{d-1} \tilde{p} \int_{0} \frac{\mathrm{d} s}{\sqrt{s}} \int_{0} \mathrm{~d} v
$$

where we only indicate the integration limit for the region of interest. We now use $E_{p}=$ $E_{\tilde{p}}+s+O\left(s^{2}\right)$, so that $E_{p}-u=s+v+O\left(s^{2}\right)$, and $\mathcal{E}_{u, \tilde{p}} \propto \sqrt{v}(1+O(v))$. Omitting proportionality factors the leading large- $t$ behaviour of (46) is

$$
\left.x_{j}^{1} \int_{0} \frac{\mathrm{d} s}{\sqrt{s}} \int_{0} \mathrm{~d} v \sqrt{v} \frac{\mathrm{e}^{\mathrm{i}(s+v) t}}{s+v} \propto x_{j}^{1} t^{-1} \quad \text { (initially free at } x^{1}=0\right) .
$$

Similarly, using (44) we get

$$
\left.\int_{0} \mathrm{~d} s \int_{0} \frac{\mathrm{d} v}{\sqrt{v}} \frac{\mathrm{e}^{\mathrm{i}(s+v) t}}{s+v} \propto t^{-\frac{1}{2}} \quad \text { (initially fixed at } x^{1}=0\right) .
$$

In both cases, the proportionality factor is a function of the mass, the temperatures, and $t_{i}, t_{j}$, $x_{i}$ and $\tilde{x}_{j}$.

The results (48) and (49) give the contribution to the leading large- $t$ approach to the steady state coming from one Wick contraction, and one choice of the member of the contracted pair where the $\Psi$ term is taken. The leading large- $t$ asymptotic is obtained by summing over every pair of fields, and for every pair, summing over the two contributions coming from taking the $\Psi$ term for either member in the pair. The contribution coming from the contraction $\left(S\left(\phi\left(t_{i}, x_{i}\right)\right), \Psi\left(t_{j}, x_{j}\right)\right)$ is of the form $J x_{j}^{1} t^{-1}$ (resp. $\left.J t^{-1 / 2}\right)$ for free (resp. fixed) initial boundary condition, where the factor $J$ depends on $x_{j}^{2, \ldots, d}, x_{i}^{2, \ldots, d}, t_{i}-t_{j}$, and on the other coordinates $x_{k}, k \neq i, j$. The factor $J$ does not depend on $x_{i}^{1}, x_{j}^{1}$ or $t_{i}+t_{j}$. Knowing this structure, we may extend this to local observables containing $x_{i}$ and $t_{i}$ derivatives. The leading power of $t$ is unchanged, unless a derivative with respect to $x_{i}^{1}$ of high enough order makes the coefficient of the leading behaviour vanish.

In conclusion, we find the following. In a long-time asymptotic analysis, the steady state (10) with (9) is reached as $t \rightarrow \infty$, and is approached with a power law in $t$ as follows:

$$
\operatorname{Tr}\left(\mathfrak{n}\left[\rho_{0}\right] \mathrm{e}^{\mathrm{i} H t} \mathcal{O} \mathrm{e}^{-\mathrm{i} H t}\right)=\langle\mathcal{O}\rangle_{\mathrm{s}}+ \begin{cases}O\left(t^{-1}\right) & \text { (initially free at } \left.x^{1}=0\right) \\ O\left(t^{-\frac{1}{2}}\right) & \text { (initially fixed (or mixed) at } \left.x^{1}=0\right)\end{cases}
$$

for any $\mathcal{O}=\prod_{i} \mathcal{O}_{i}\left(t_{i}, x_{i}\right)$ that is a product of local observables. Further, the coefficient in the initially free case decreases like $O(R)$ as $R \rightarrow 0$, where $R=\max \left(\left\{\left|x_{i}^{1}\right|: i\right\}\right)$ is the largest distance of a local observable to the connection hypersurface:

$$
\left.\lim _{t \rightarrow \infty} t\left(\operatorname{Tr}\left(\mathfrak{n}\left[\rho_{0}\right] \mathrm{e}^{\mathrm{i} H t} \mathcal{O} \mathrm{e}^{-\mathrm{i} H t}\right)-\langle\mathcal{O}\rangle_{\mathrm{s}}\right) \stackrel{R \rightarrow 0}{=} O(R) \quad \text { (initially free at } x^{1}=0\right) .
$$

From this we make the following final observation.

4. For free initial boundary conditions at $x^{1}=0$, the approach to the steady state, proportional to $t^{-1}$, is generically faster than that for fixed or mixed initial boundary conditions, proportional to $t^{-1 / 2}$. Again this may be explained as in Point 2 above, from the intuition that with free conditions, it is easier for the fields to reach their steady state value. They do so instantaneously at $x^{1}=0$, and faster the closer they are to the hypersurface $x^{1}=0$. 
It is important to emphasize that equation (50) gives the leading time dependence for generic observables. However, depending on the observable being considered, the leading order contribution may vanish giving rise to a faster approach. As we will show in the next section, this occurs for the energy current and the energy density. Nonetheless, the approach to the steady state remains a power-law in time and is fully described by the above formalism.

An example of an observable whose leading time dependence is of the generic form is the two-point function of the fundamental field $\phi(x)$. For instance, an analysis as above, keeping track of the precise coefficients, give for initially fixed boundary conditions,

$$
\langle\phi(x, t) \phi(y, t)\rangle-\langle\phi(x, t) \phi(y, t)\rangle_{\mathrm{s}}=\frac{1}{\sqrt{\pi m t}} \frac{\sinh \frac{\left(\beta_{\mathrm{L}}+\beta_{\mathrm{R}}\right) m}{2}}{\sinh \frac{\beta_{\mathrm{L}} m}{2} \sinh \frac{\beta_{\mathrm{R}} m}{2}}\left(1+O\left(t^{-1}\right)\right) .
$$

\subsection{Time-evolution of the average energy current and energy density}

In the above analysis we have discussed the time-evolution of local operators. In this section we discuss the implications for the time-evolution of the average energy current. For simplicity we consider the case with $d=1$ and $m \neq 0$. As we discuss more fully in Appendix D, the long time approach to the NESS for fixed initial boundary conditions is given by

$$
\left\langle T^{01}\right\rangle=\left\langle T^{01}\right\rangle_{s}-\delta\left(\beta_{\mathrm{L}}, \beta_{R}\right),
$$

where $\delta\left(\beta_{\mathrm{L}}, \beta_{R}\right) \equiv \delta\left(\beta_{\mathrm{L}}\right)-\delta\left(\beta_{R}\right)$ and

$$
\delta(\beta)=\frac{3 \beta m}{64 \pi \sinh ^{2}(\beta m / 2)}\left(1+\frac{2}{3} \frac{\left(1-\mathrm{e}^{-\beta m}\right)}{\beta m} \sin (2 m t)\right) t^{-2}+O\left(t^{-3}\right) .
$$

The approach to the steady state is therefore governed by a $t^{-2}$ power-law with an oscillatory contribution. This dependence is confirmed numerically in Fig. 3; for details of the numerical approach see Appendix E. The corresponding evolution for free initial boundary conditions is shown in Fig. 4 and is compatible with a $t^{-1}$ approach. Plots of the time evolution of the local energy density $\left\langle T^{00}\right\rangle$ are shown in Figs. 5 and 6 . Plots of the time evolution of the spatial profile are given in Fig. 7 .

From Figs. 4-6, we observe that, for initially free boundary conditions, both the energy current and energy density approach the steady state with the generic (i.e. the slowest allowed) power law $\propto t^{-1}$. On the other hand, for initially fixed boundary conditions, the approach is, in both cases, faster than the generic behaviour $\propto t^{-1 / 2}$. In all cases, the approach is modulated by oscillations, which appear to be stronger for the energy current than for the energy density. It is also interesting to note that for the energy current, initially fixed boundary conditions lead to a faster approach to the steady state than initially free boundary conditions, in contrast with the generic case. This could be understood by the fact, emphasized above, that initially fixed (free) boundary conditions for the fundamental field $\phi$ at $x^{1}=0$ correspond to initially free (fixed) boundary conditions for the longitudinal derivative $\partial_{1} \phi$ at $x^{1}=0$. This naturally affects the longitudinal current, which reaches its steady state value more easily for initial conditions that are free on the longitudinal derivative $\partial_{1} \phi$, hence fixed on $\phi$.

From Fig. 7 we observe the monotonic spacial behaviour of the energy density, interpolating between the left and right reservoirs; and the monotonicity of the energy current in the transition 

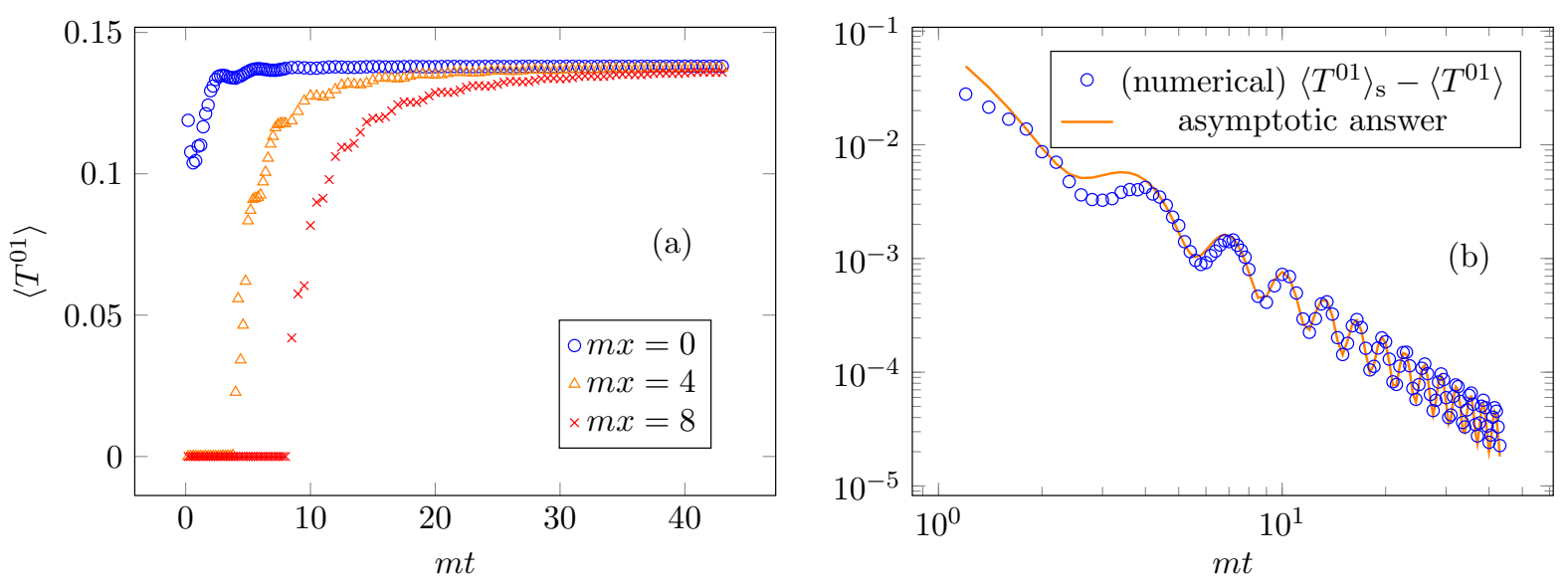

Figure 3: Numerical solutions in $d=1$ with $T=m=1$ and fixed pre-quench boundary conditions. (a) Time-evolution of the energy current $\left\langle T^{01}\right\rangle$ at different distances $x$ from the connection hypersurface, showing the approach to the steady state $\left\langle T^{01}\right\rangle_{s}$ at late times. (b) Fit to the theoretical prediction (53) showing the oscillatory behavior and the $t^{-2}$ power-law.

regions, interpolating between the reservoirs and the central region (where the steady-state forms). We also see the light-wedge effect, by which the average values of observables are unchanged beyond the light wedge $\left|x^{1}\right|>t$, in agreement with the first line of (41).

\subsection{Semiclassical analysis of energy flow evolution and transition regions}

We have derived the properties of the steady state, including exact expressions for the operator $\phi(x, t)$ and therefore for any observable such as $T^{\mu \nu}(x, t)$. It is also instructive to understand how the steady state emerges in the simultaneous limit of large time and far from the connection region, by explicitly evaluating expectation values of $T^{01}(x, t)$ using a semiclassical analysis. This analysis is valid asymptotically in the limit where $x^{1}, t \rightarrow \infty$ with $x^{1} / t$ fixed. Note that this is outside of the regime of validity of Eq. (43). Here we perform this calculation in the massless limit $m=0$ for simplicity, but the procedure straightforwardly generalizes to the massive case.

The calculation is based on semiclassical insight into the evolution of $\left\langle T^{\mu \nu}\right\rangle$. A similar semiclassical approach was used in $[60,51]$. As this is a free theory, let us begin by considering only the modes with momentum $p$ in the left bath. Without loss of generality, we may assume that $x^{1}>0$. All of the bosons with momentum $p$ move at the (group) velocity $p^{1} /|p| \equiv v_{p}$ in the $x^{1}$-direction. On the world-surface $x^{1} / t=v_{p_{0}}$, only modes at velocities $v_{p}>v_{p_{0}}$ will contribute from the left as other modes are not fast enough to reach the world-surface. On the other hand, from the right, all modes at velocities $v_{p}<v_{p_{0}}$ will contribute. This includes all modes from the right with negative velocity, and also some with positive velocity, going in the "wrong" direction: those that are slow enough to cross the world-surface. But modes from the right with opposite velocities give exactly opposite contributions to the current, hence cancel out. That is, the heat flow is given by counting all of the momentum modes coming from opposite baths at velocities $\left|v_{p}\right|<v_{p_{0}}$ : positive velocity from the left, negative from the right. An integral, mimicking the 

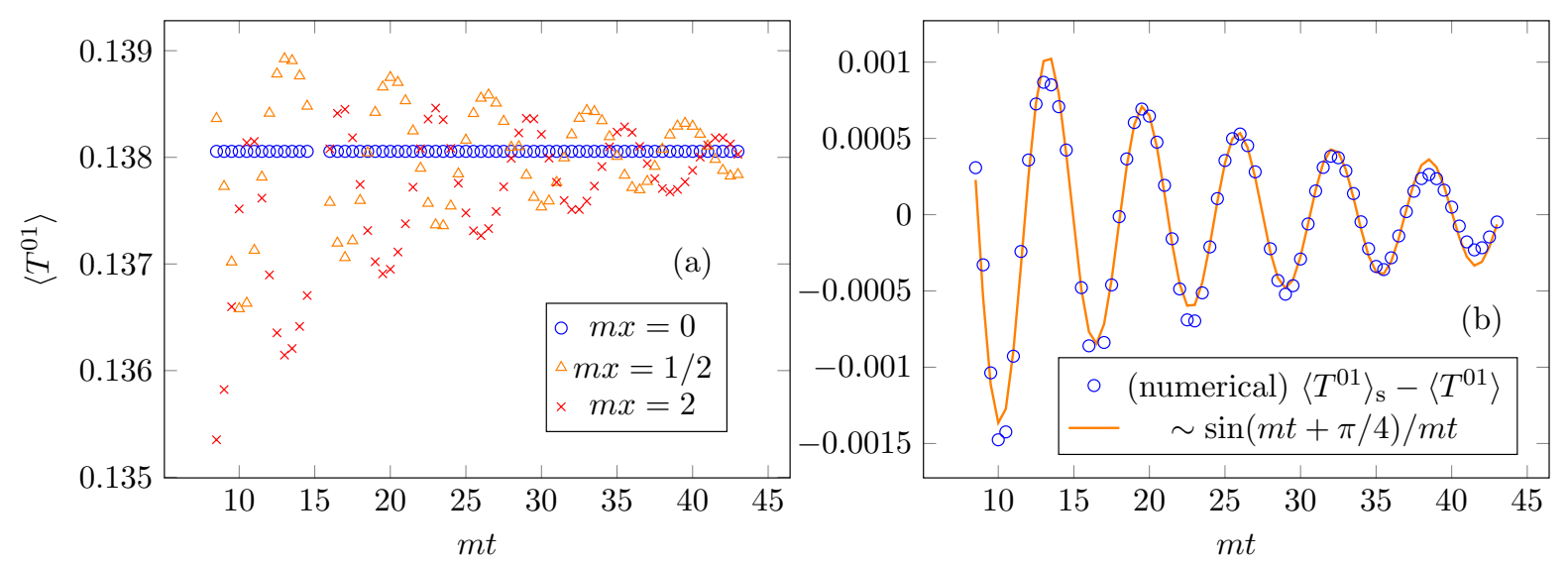

Figure 4: Numerical solutions in $d=1$ with $T=m=1$ and free pre-quench boundary conditions. (a) Time-evolution of the energy current $\left\langle T^{01}\right\rangle$ at different distances $x$ from the connection hypersurface, showing the approach to the steady state $\left\langle T^{01}\right\rangle_{s}$ at late times, and the instantaneous approach at $x=0$. (b) Case $x=1 / 2$. Fit to the function $\sin (m t+\pi / 4) / m t$, giving a proportionality constant of 0.01395 .
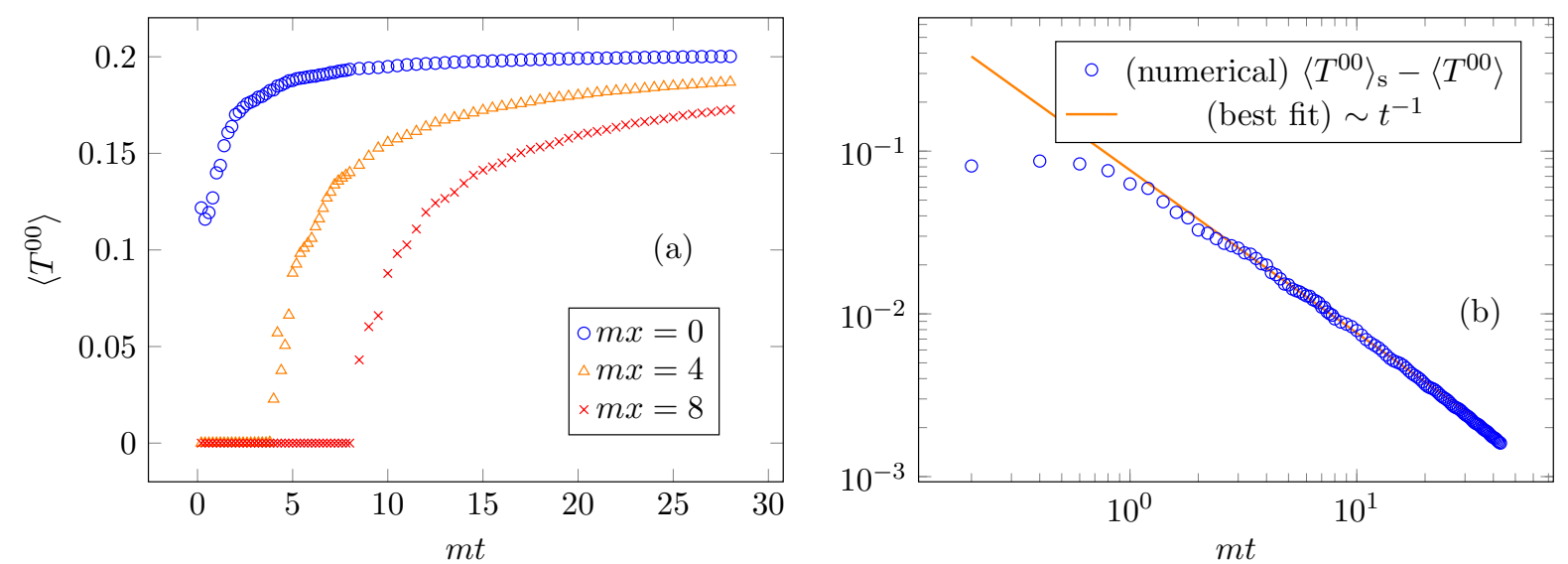

Figure 5: Numerical solutions in $d=1$ with $T=m=1$ and fixed pre-quench boundary conditions. (a) Time-evolution of the energy density $\left\langle T^{00}\right\rangle$ at different distances $x$ from the connection hypersurface, showing the approach to the steady state $\left\langle T^{00}\right\rangle_{s}$ at late times. (b) The asymptotics are compatible with a $t^{-1}$ power-law approach. Here, the case $x=1 / 2$ is shown, where a proportionality constant of 0.07639 is found. 

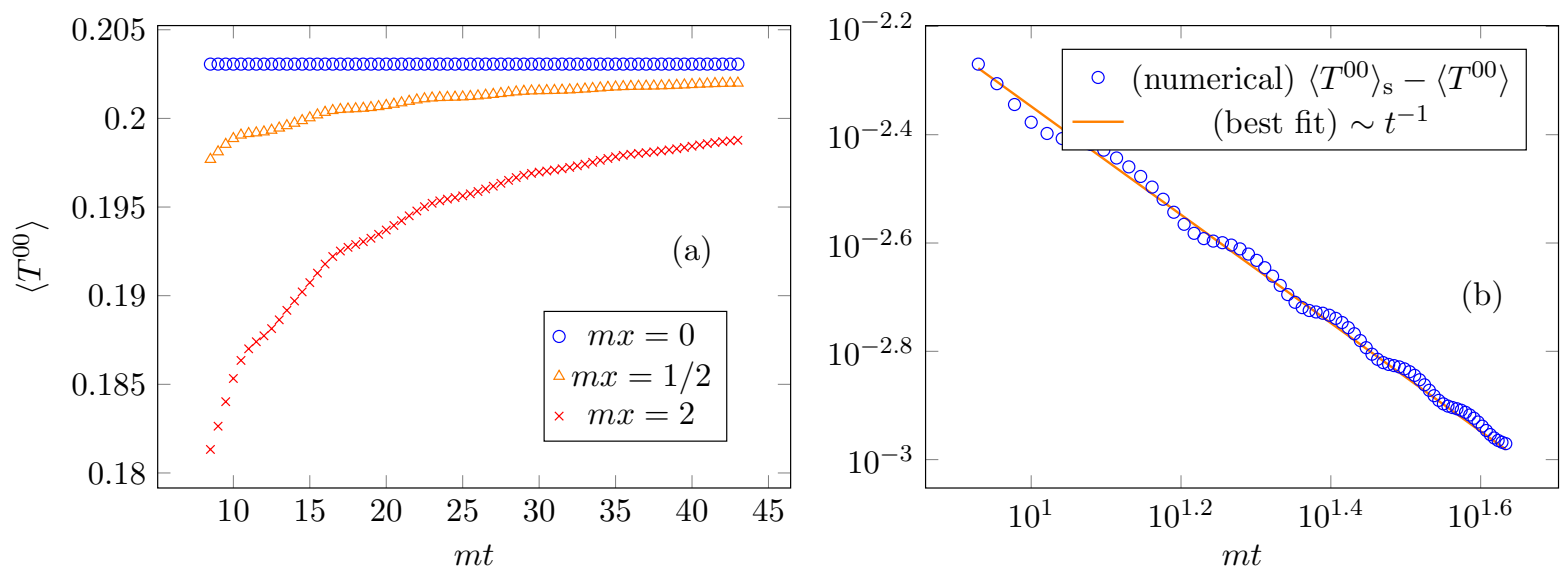

Figure 6: Numerical solutions in $d=1$ with $T=m=1$ and free pre-quench boundary conditions. (a) Time-evolution of the energy density $\left\langle T^{00}\right\rangle$ at different distances $x$ from the connection hypersurface, showing the approach to the steady state $\left\langle T^{00}\right\rangle_{s}$ at late times, and the instantaneous approach at $x=0$. (b) The asymptotics are compatible with a $t^{-1}$ power-law approach (up to small oscillations). Here, the case $x=1 / 2$ is shown, where a proportionality constant of 0.0449 is found.
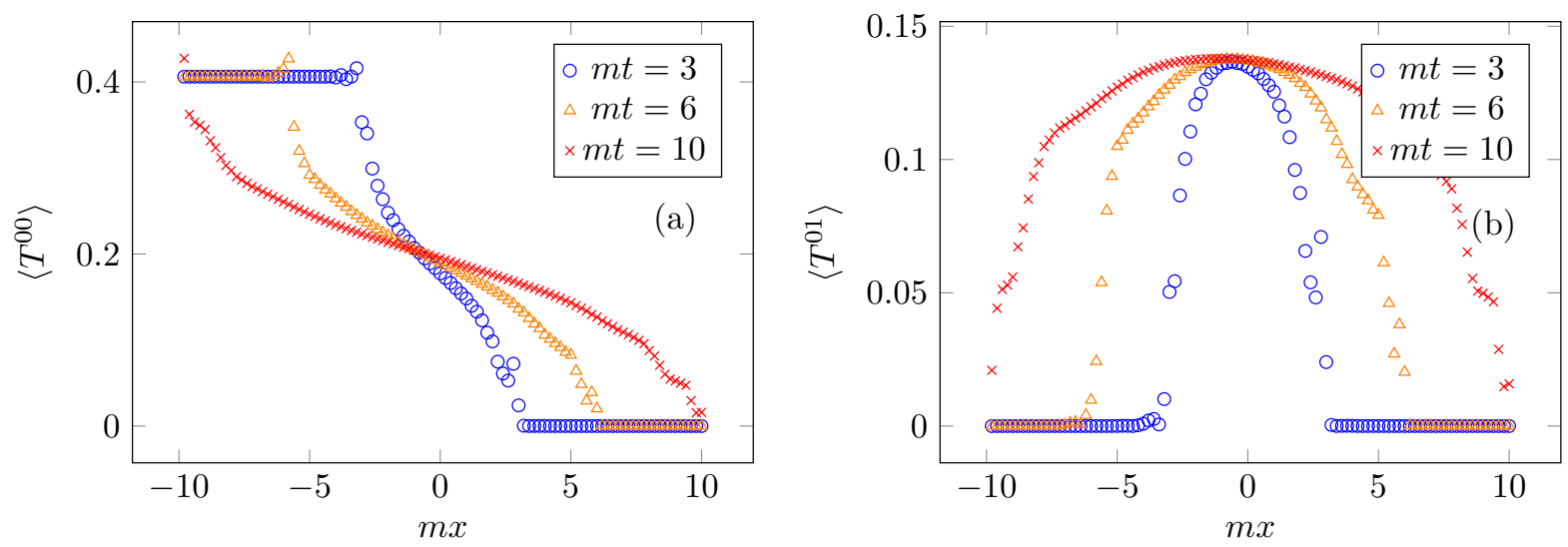

Figure 7: Numerical solutions in $d=1$ with $T=m=1$ and fixed pre-quench boundary conditions. Spatial profiles of (a) the local energy density $\left\langle T^{00}(x)\right\rangle$ and (b) the energy current $\left\langle T^{01}(x)\right\rangle$ for different times. 
exact steady-state calculation described in Appendix A, then gives us

$$
\begin{aligned}
\left\langle T^{01}\right\rangle & =(d-1) C_{\mathrm{L}, \mathrm{R}}\left(T_{\mathrm{L}}^{d+1}-T_{\mathrm{R}}^{d+1}\right) \int_{0}^{\theta_{0}} \mathrm{~d} \sin \theta_{1} \sin ^{d-2} \theta_{1} \\
& =C_{\mathrm{L}, \mathrm{R}}\left(T_{\mathrm{L}}^{d+1}-T_{\mathrm{R}}^{d+1}\right)\left(1-\frac{\left(x^{1}\right)^{2}}{t^{2}}\right)^{(d-1) / 2},
\end{aligned}
$$

where the normalization constants $C_{\mathrm{L}, \mathrm{R}}$ are given in Appendix $\mathrm{A}$, and $C_{\mathrm{L}}=C_{\mathrm{R}}$ in the massless case. It is readily seen that the same expression holds for $x^{1} / t<0$. The above semiclassical argument may be justified from the exact quantum computation; see Appendix H.

This semiclassical analysis emphasizes the fact that, as suggested by Fig. 1, the transition region, interpolating between the baths and the steady-state region, is large in $d>1$ and presents a smoothly interpolating average current. We expect this to hold for other observables as well, such as the energy density. The intuition is that modes of various velocities, which do not interact with each other, arrive, at any given point, at different times, slowly building up the steady state and producing large transients and transition regions. This is in strong contrast with the $d=1$ results, where sharp transitions are found between the asymptotic baths and the steady state, due to the one-dimensional relativistic dispersion relation giving rise to a single velocity (the velocity of light) for all momenta. The present situation is very different: the transition region is large on macroscopic scales.

The above semiclassical analysis is based on a picture of freely-propagating particles. The evolution of $\left\langle T^{01}\right\rangle$ in a strongly interacting theory is inherently different. With interactions, the time evolution of averages is quickly governed by hydrodynamics, as interactions give rise to local thermalization effects. Using these insights, it was found in [40] that at leading order as $x^{1} / t \rightarrow \infty,\left\langle T^{01}\right\rangle$ is a piecewise constant function with two jumps, corresponding to the propagation of "shock waves".

\section{Discussion: effects of interactions}

As we stressed in the introduction, in contrast to the one-dimensional case, the steady state in the massless Klein-Gordon theory in $d>1$ is different from that found in interacting higherdimensional CFT [40]. This difference is due to the infinite number of local conserved charges in the Klein-Gordon theory. These conserved charges imply that the occupation numbers $N_{p}=A_{p}^{\dagger} A_{p}$ are conserved for every momentum $p$, which allows for the momentum modes to be thermalized independently. In a generic interacting QFT, one does not find this large number of conserved charges. Generically, one does not expect any conserved quantities other than those associated with space-time symmetries: the total energy $H$, the total momentum $P^{i}$, and the boost generators. By time and translation invariance of the steady state density matrix, the boost generator cannot contribute, so that in this case the non-equilibrium steady state is given by a boosted thermal state [40], rather than a collection of independently thermalized propagating modes. This indicates that in, contrast to the situation in one-dimension where the free-field and boost descriptions coincide, in higher-dimensions, the free-field NESS is unstable to perturbations. It would be very interesting to understand the detailed evolution between the non-interacting and interacting regimes. One possibility in a weakly interacting system is that 
the non-equilibrium behavior is described by the free-field limit at early times, before crossing over to the hydrodynamic regime. In this scenario, the system would 'pre-thermalize' as an ensemble of independent and approximately conserved modes, followed by a more rapid onset towards hydrodynamics. In this picture, the pre-thermalization is approached as a power law in time, and would not be seen unless the interactions are sufficiently weak so that the hydrodynamic onset occurs at late enough times. An estimate of the timescale for "hydronization" may be obtained by dimensional analysis and scaling arguments. For the case of " $\phi$ " interactions:

$$
H_{\mathrm{int}}=\frac{\lambda}{4 !} \int \mathrm{d}^{d} x: \phi(x, t)^{4}:
$$

one obtains

$$
t_{\text {hydro }} \sim T^{5-2 d} \lambda^{-2} .
$$

This formula is only valid when the dimensionless combination $\lambda T^{d-3} \ll 1$, corresponding to the limit where kinetic theory is valid; this is at large temperatures if $d<3$ (the coupling is relevant and the free UV fixed point is nearby). At the marginal dimension $d=3$ one finds the strongly-coupled, hydrodynamic form $t_{\text {hydro }} \sim 1 / T$, up to a factor of $\lambda^{-2}$. It would interesting to develop kinetic theory approaches to investigate this crossover.

\section{Conclusion}

We have considered the non-equilibrium dynamics of the Klein-Gordon model following a local quench in arbitrary dimension. We have adopted the partitioning approach in which two independently thermalized halves are brought into contact and are allowed to evolve unitarily. We have demonstrated that the steady state density matrix generically contains non-local contributions, away from the massless limit in one-dimension. This results in a power-law approach to the steady state, where the exponent depends on the pre-quench boundary conditions on the connection hypersurface. We provide exact results for the steady state energy current, the energy density and the scaled cummulants of the energy transfer. The statistics of the energy transfer are described by a continuum of independent Poisson processes, where the weights are analytically determined. When specialized to zero mass and one dimension, the results found here reproduce the known results from conformal field theory [33, 34] at central charge $c=1$. Some of the results are also in correspondence with those obtained in free fermion models in higher dimensions [51]. However, in contrast to the behavior of massless free-fields in one-dimension, which have a direct connection to the generic results of $1+1 \mathrm{CFT}$, the free-field limit does not capture the non-equilibrium behavior of generic CFTs in higher dimensions [40]. This is a direct consequence of the anomalous behavior of free-fields due to the proliferation of conservation laws. Many of these conservation laws are explicitly broken in the presence of interactions and cannot contribute to the generic non-equilibrium density matrix. It is interesting to note that in the special case where $d=1$ and $m=0$ these additional contributions drop out from the free-field density matrix so that the free-field and CFT descriptions coincide. It would be very illuminating to understand the evolution between the free-field limit and the interacting regime in more detail for higher dimensional situations. 


\section{Acknowledgements}

B.D. thanks the informal CFT discussion group at King's for comments on this work, Édouard Boulat, Pasquale Calabrese, Mario Collura, Márton Kormos, Gabriele Martelloni, Hubert Saleur and Jacopo Viti for discussions, and Université Paris Diderot, where part of this work was done, for financial support through a visiting professorship. A.L. is supported by the Smith Family Graduate Science and Engineering Fellowship, and would like to thank the Perimeter Institute of Theoretical Physics for hospitality as this work was in progress. Research at Perimeter Institute is supported by the Government of Canada through Industry Canada and by the Province of Ontario through the Ministry of Economic Development \& Innovation. This work was further supported in part by a VICI grant of the Netherlands Organization for Scientific Research (NWO), by the Netherlands Organization for Scientific Research/Ministry of Science and Education (NWO/OCW) and by the Foundation for Research into Fundamental Matter (FOM). MJB acknowledges helpful discussions with Joel Moore and thanks the Thomas Young Center.

\section{A Energy current and energy density}

Here we compute the momentum current in the steady state

$$
\left\langle T^{01}\right\rangle_{\mathrm{s}}=\int \frac{\mathrm{d}^{d} p}{(2 \pi)^{d}}\left|p^{1}\right|\left(\frac{\Theta\left(p^{1}\right)}{\mathrm{e}^{\beta_{\mathrm{L}} E_{p}}-1}-\frac{\Theta\left(-p^{1}\right)}{\mathrm{e}^{\beta_{\mathrm{R}} E_{p}}-1}\right) .
$$

To evaluate the $p_{1}$ integral we separate it into a sum of integrals over the positive and negative real line, we find

$$
\left\langle T^{01}\right\rangle=C_{\mathrm{L}} T_{\mathrm{L}}^{d+1}-C_{\mathrm{R}} T_{\mathrm{R}}^{d+1}, \quad\left\langle T^{00}\right\rangle=A_{\mathrm{L}} T_{\mathrm{L}}^{d+1}+A_{\mathrm{R}} T_{\mathrm{R}}^{d+1}
$$

where

$$
\begin{aligned}
& C_{\mathrm{L}, \mathrm{R}}=\int_{p_{1}>0} \frac{\mathrm{d}^{d} p}{(2 \pi)^{d}} \frac{p_{1}}{\mathrm{e}^{\sqrt{p^{2}+m_{\mathrm{L}, \mathrm{R}}^{2}}}-1}=d ! \zeta_{m_{\mathrm{L}, \mathrm{R}}}(d+1) \int_{\substack{|a|=1 \\
a_{1}>0}} \frac{\mathrm{d}^{d-1} a}{(2 \pi)^{d}} a_{1} \\
& A_{\mathrm{L}, \mathrm{R}}=\int_{p_{1}>0} \frac{\mathrm{d}^{d} p}{(2 \pi)^{d}} \frac{\sqrt{p^{2}+m_{\mathrm{L}, \mathrm{R}}^{2}}}{\mathrm{e}^{\sqrt{p^{2}+m_{\mathrm{L}, \mathrm{R}}^{2}}-1}}=d ! \tilde{\zeta}_{m_{\mathrm{L}, \mathrm{R}}}(d+1) \int_{\substack{|a|=1 \\
a_{1}>0}} \frac{\mathrm{d}^{d-1} a}{(2 \pi)^{d}}
\end{aligned}
$$

with $m_{\mathrm{L}, \mathrm{R}}:=m / T_{\mathrm{L}, \mathrm{R}}$ and

$$
\zeta_{b}(d):=\frac{1}{\Gamma(d)} \int_{0}^{\infty} \mathrm{d} p \frac{p^{d-1}}{\mathrm{e}^{\sqrt{p^{2}+b^{2}}}-1}, \quad \tilde{\zeta}_{b}(d):=\frac{1}{\Gamma(d)} \int_{0}^{\infty} \mathrm{d} p \frac{p^{d-2} \sqrt{p^{2}+b^{2}}}{\mathrm{e}^{\sqrt{p^{2}+b^{2}}}-1} .
$$

We note that $\zeta_{0}(d)=\tilde{\zeta}_{0}(d)=\zeta(d)$ is Riemann's zeta function. In order to evaluate the angular integrals, one may use the $d-1$ angles $\theta_{1}, \ldots, \theta_{d-1}$ on the hypersphere, with $a_{1}=\cos \theta_{1}$. The total surface of the $(d-1)$-dimensional unit sphere (embedded in $d$ space dimensions) is

$$
\int_{-\pi}^{\pi} \mathrm{d} \theta_{d-1} \int_{0}^{\pi} \mathrm{d} \theta_{d-2} \cdots \mathrm{d} \theta_{1} \sin \theta_{d-2} \sin ^{2} \theta_{d-3} \cdots \sin ^{d-2} \theta_{1}=\frac{2 \pi^{d / 2}}{\Gamma(d / 2)} .
$$


Hence we directly have

$$
A_{\mathrm{L}, \mathrm{R}}=\frac{d \Gamma\left(\frac{d+1}{2}\right) \tilde{\zeta}_{m_{\mathrm{L}, \mathrm{R}}}(d+1)}{2 \pi^{(d+1) / 2}}
$$

and we may calculate

$$
\begin{aligned}
\int_{\substack{|a|=1 \\
a_{1}>0}} \frac{\mathrm{d}^{d-1} a}{(2 \pi)^{d}} a_{1} & =\frac{1}{(2 \pi)^{d}} \int_{-\pi}^{\pi} \mathrm{d} \theta_{d-1} \int_{0}^{\pi} \mathrm{d} \theta_{d-2} \cdots \int_{0}^{\pi / 2} \mathrm{~d} \theta_{1} \sin \theta_{d-2} \sin ^{2} \theta_{d-3} \cdots \sin ^{d-2} \theta_{1} \cos \theta_{1} \\
& =\frac{1}{(2 \pi)^{d}} \frac{2 \pi^{(d-1) / 2}}{\Gamma((d-1) / 2)} \int_{0}^{\pi / 2} \mathrm{~d} \theta_{1} \cos \theta_{1} \sin ^{d-2} \theta_{1} \\
& =\frac{1}{(2 \pi)^{d}} \frac{2 \pi^{(d-1) / 2}}{\Gamma((d-1) / 2)} \int_{0}^{1} \mathrm{~d} v v^{d-2} \\
& =\frac{1}{(2 \pi)^{d}} \frac{2 \pi^{(d-1) / 2}}{\Gamma((d-1) / 2)} \frac{1}{d-1}
\end{aligned}
$$

which gives

$$
C_{\mathrm{L}, \mathrm{R}}=\frac{d \Gamma\left(\frac{d}{2}\right) \zeta_{m_{\mathrm{L}, \mathrm{R}}}(d+1)}{2 \pi^{d / 2+1}} .
$$

In $d=1$, using $\zeta(2)=\pi^{2} / 6$ and $\Gamma(1 / 2)=\sqrt{\pi}$, we find $A=C=\pi / 12$ as it should for a one-dimensional CFT of central charge 1 .

\section{B Steady-state density matrix in terms of local fields}

Here we determine the steady state density matrix $\rho_{\mathrm{s}}=\exp -\widehat{W}$ where

$$
\widehat{W}=\frac{\beta_{\mathrm{L}}+\beta_{\mathrm{R}}}{2} H+\frac{\beta_{\mathrm{L}}-\beta_{\mathrm{R}}}{2}\left(P_{1}+\widehat{Q}\right) .
$$

First note that

$$
\left.\widehat{(} P_{1}+\widehat{Q}\right)=\int D p \operatorname{sign}\left(p^{1}\right) E_{p} A_{p}^{\dagger} A_{p}
$$

The inversion of (4) gives

$$
A_{p}=\int \mathrm{d}^{d} x \mathrm{e}^{-\mathrm{i} p \cdot x}\left(E_{p} \phi(x)+\mathrm{i} \pi(x)\right)
$$

which we insert into (61):

$\left.\widehat{(} P_{1}+\widehat{Q}\right)=\int \mathrm{d}^{d} x \mathrm{~d}^{d} y\left[: \phi(x) \phi(y): Q^{\prime}(x-y)+: \pi(x) \pi(y): Q^{\prime \prime}(x-y)+: \phi(x) \pi(y): Q^{\prime \prime \prime}(x-y)\right]$

where

$$
\begin{aligned}
Q^{\prime}(x) & =\int \frac{\mathrm{d}^{d} p}{2(2 \pi)^{d}} \operatorname{sign}\left(p^{1}\right) E_{p}^{2} \mathrm{e}^{\mathrm{i} p \cdot x} \\
Q^{\prime \prime}(x) & =\int \frac{\mathrm{d}^{d} p}{2(2 \pi)^{d}} \operatorname{sign}\left(p^{1}\right) \mathrm{e}^{\mathrm{i} p \cdot x} \\
Q^{\prime \prime \prime}(x) & =i \int \frac{\mathrm{d}^{d} p}{(2 \pi)^{d}} \operatorname{sign}\left(p^{1}\right) E_{p} \mathrm{e}^{\mathrm{i} p \cdot x} .
\end{aligned}
$$


Clearly $Q^{\prime}(x)$ and $Q^{\prime \prime}(x)$ are odd under $x \mapsto-x$, hence the first two terms in (63) vanish. On the other hand we can write

$$
\begin{aligned}
Q^{\prime \prime \prime}(x) & =\mathrm{i} \int \frac{\mathrm{d}^{d-1} \tilde{p}}{(2 \pi)^{d-1}} \mathrm{e}^{\mathrm{i} \tilde{p} \cdot \tilde{x}}\left(\int \frac{\mathrm{d} p^{1}}{2 \pi} p^{1} \mathrm{e}^{\mathrm{i} p^{1} x^{1}}+\int \frac{\mathrm{d} p^{1}}{2 \pi} \operatorname{sign}\left(p^{1}\right)\left(E_{p}-\left|p^{1}\right|\right) \mathrm{e}^{\mathrm{i} p^{1} x^{1}}\right) \\
& =\partial_{1} \delta^{(d)}(x)+\mathrm{i} \int \frac{\mathrm{d}^{d-1} \tilde{p}}{(2 \pi)^{d-1}} \mathrm{e}^{\mathrm{i} \tilde{p} \cdot \tilde{x}}\left(\int \frac{\mathrm{d} p^{1}}{2 \pi} \operatorname{sign}\left(p^{1}\right)\left(E_{p}-\left|p^{1}\right|\right) \mathrm{e}^{\mathrm{i} p^{1} x^{1}}\right)
\end{aligned}
$$

where the tilde-variables represent transverse coordinates. The first term on the last line gives rise to the $P_{1}$ contribution in (29). For the second term, let us concentrate on the $p^{1}$-integral, which we write as

$$
\int_{0}^{\infty} \frac{\mathrm{d} p^{1}}{2 \pi}\left(E_{p}-p^{1}\right)\left(\mathrm{e}^{\mathrm{i} p^{1} x^{1}}-\mathrm{e}^{-\mathrm{i} p^{1} x^{1}}\right)
$$

The large- $p^{1}$ behavior of $E_{p}-p^{1}$ is vanishing, hence the integral is convergent and we may shift the contours. Let us assume $x^{1}>0$; this is sufficient as the integral is odd under $x^{1} \mapsto-x^{1}$. Then for the terms proportional to $\mathrm{e}^{ \pm \mathrm{i} p^{1} x^{1}}$ we rotate the contours towards the positive/negative imaginary axis, $p^{1}= \pm \mathrm{i} \ell$. The part proportional to $p^{1}$ cancels out, hence we obtain

$$
\mathrm{i} \int_{0}^{\infty} \frac{\mathrm{d} \ell}{2 \pi}\left(\sqrt{|\tilde{p}|^{2}+m^{2}-\ell^{2}+\mathrm{i} \mathbf{0}}+\sqrt{|\tilde{p}|^{2}+m^{2}-\ell^{2}-\mathrm{i} \mathbf{0}}\right) \mathrm{e}^{-x^{1} \ell}=\frac{\mathrm{i}}{\pi} \int_{0}^{E_{\tilde{p}}} \mathrm{~d} \ell \mathcal{E}_{\ell, \tilde{p}} \mathrm{e}^{-x^{1} \ell} .
$$

This gives (31).

\section{Time evolution of fields}

Here we compute the explicit form of the time evolved fields $\phi(x, t)$ and $\pi(x, t)$ in the $B$ representation. For clarity, let us momentarily use the hat symbol in order to differentiate operators from fields. One can show that if $\widehat{\phi}(x, t)$ and $\widehat{\pi}(x, t)$ are operators obeying the canonical commutation relations (2) (in any representation) and satisfying the equations of motion (40), then the functions

$$
\begin{aligned}
\phi(x, t) & =-\mathrm{i} \int d^{d} y([\widehat{\phi}(x, t), \widehat{\pi}(y)] \phi(y)-[\widehat{\phi}(x, t), \widehat{\phi}(y)] \pi(y)) \\
\pi(x, t) & =-\mathrm{i} \int d^{d} y([\widehat{\pi}(x, t), \widehat{\pi}(y)] \phi(y)-[\widehat{\pi}(x, t), \widehat{\phi}(y)] \pi(y)),
\end{aligned}
$$

are solutions to the equations of motion (40) with initial conditions $\phi(0, x)=\phi(x)$ and $\pi(0, x)=$ $\pi(x)$. If we replace $\phi(y)$ and $\pi(y)$ by the $B$-representation (35), then by construction we have found operators $\widehat{\phi}(x, t)$ and $\widehat{\pi}(x, t)$ in the $B$-representation.

Using the $A$-representation, the operators $\widehat{\phi}(x, t)$ and $\widehat{\pi}(x, t)$ are given by (34), and we may explicitly evaluate the commutators:

$$
\begin{aligned}
{[\widehat{\phi}(x, t), \widehat{\phi}(y)] } & =-\mathrm{i} \int D p 2 \sin \left(E_{p} t\right) \mathrm{e}^{\mathrm{i} p \cdot(x-y)} \\
{[\widehat{\phi}(x, t), \widehat{\pi}(y)] } & =\mathrm{i} \int D p 2 E_{p} \cos \left(E_{p} t\right) \mathrm{e}^{\mathrm{i} p \cdot(x-y)} \\
{[\widehat{\pi}(x, t), \widehat{\phi}(y)] } & =-\mathrm{i} \int D p 2 E_{p} \cos \left(E_{p} t\right) \mathrm{e}^{\mathrm{i} p \cdot(x-y)} \\
{[\widehat{\pi}(x, t), \widehat{\pi}(y)] } & =-\mathrm{i} \int D p 2 E_{p}^{2} \sin \left(E_{p} t\right) \mathrm{e}^{\mathrm{i} p \cdot(x-y)} .
\end{aligned}
$$


Putting (68) and (35) in (67) and evaluating the resulting integrals, we find

$$
\widehat{\phi}(x, t)=\int D p B_{p} \int \frac{\mathrm{dq}}{2 \pi} U\left(p^{1}, \mathrm{q}\right) \mathrm{e}^{\mathrm{iq} x^{1}+\mathrm{i} \tilde{p} \cdot \tilde{x}}\left(a_{\mathrm{q}, p}^{+} \mathrm{e}^{-\mathrm{i} E_{\mathrm{q}, \tilde{p}} t}+a_{\mathrm{q}, p}^{-} e^{\mathrm{i} E_{\mathrm{q}, \tilde{p}} t}\right)+\text { h.c. }
$$

where

$$
a_{\mathrm{q}, p}^{ \pm}=\frac{1}{2}\left(1 \pm \frac{E_{p}}{E_{\mathrm{q}, \tilde{p}}}\right), \quad E_{\mathrm{q}, \tilde{p}}=\sqrt{|\tilde{p}|^{2}+m^{2}+\mathrm{q}^{2}}
$$

and

$$
U(\mathrm{p}, \mathrm{q})= \begin{cases}\frac{\mathrm{i}}{\mathrm{q}-\mathrm{p}+\mathrm{i} 0}+\frac{\mathrm{i}}{\mathrm{q}+\mathrm{p}+\mathrm{i} 0} & (\mathrm{p}>0) \\ \frac{-\mathrm{i}}{\mathrm{q}-\mathrm{p}-\mathrm{i} 0}+\frac{-\mathrm{i}}{\mathrm{q}+\mathrm{p}-\mathrm{i} 0} & (\mathrm{p}<0) .\end{cases}
$$

Using (69), we may evaluate in the $B$-representation the operator $\widehat{\pi}(x, t)$ and any normal-ordered products of $\widehat{\phi}(x, t)$ and $\widehat{\pi}(x, t)$ and their derivatives. For the rest of this calculation we omit the hat symbol for field-operators.

Expression (69) can be further simplified by contour deformations. Consider the q integral in (69), omitting the factor $\mathrm{e}^{\mathrm{i} \tilde{p} \cdot \tilde{x}}$. By a change of variable, it can be written as

$$
\int_{0}^{\infty} \frac{\mathrm{dq}}{2 \pi}\left(U\left(p^{1}, \mathrm{q}\right) \mathrm{e}^{\mathrm{iq} x^{1}}+U\left(p^{1},-\mathrm{q}\right) \mathrm{e}^{-\mathrm{iq} x^{1}}\right)\left(a_{\mathrm{q}, p}^{+} \mathrm{e}^{-\mathrm{i} E_{\mathrm{q}, \tilde{p}} t}+a_{\mathrm{q}, p}^{-} \mathrm{e}^{\mathrm{i} E_{\mathrm{q}, \tilde{p}} t}\right)
$$

We deform the q-contour by rotating either to $(0, \mathrm{i} \infty)$ or $(0,-\mathrm{i} \infty)$. The direction towards which we deform is determined by the values of $x^{1}$ and $t$. We note that at $\tilde{p}$ fixed, using the fact that $E_{\mathrm{q}, \tilde{p}} \sim \mathrm{q}$, the large-q oscillating factors occur in four terms and are of the form $\mathrm{e}^{\mathrm{iq} x^{1}-\mathrm{iq} t}$, $\mathrm{e}^{\mathrm{iq} x^{1}+\mathrm{iq} t}, \mathrm{e}^{-\mathrm{iq} x^{1}-\mathrm{iq} t}$ and $\mathrm{e}^{-\mathrm{iq} x^{1}+\mathrm{iq} t}$. Hence, in order that no contribution at infinity be present upon contour deformation, we deform the q-contour towards the positive $(+)$ or negative $(-)$ imaginary direction as follows, respectively for each of the four terms (with $t>0$ ):

$$
\begin{array}{rccccc} 
& & \mathrm{e}^{\mathrm{iq} x^{1}-\mathrm{iq} t} & \mathrm{e}^{\mathrm{iq} x^{1}+\mathrm{iq} t} & \mathrm{e}^{-\mathrm{iq} x^{1}-\mathrm{iq} t} & \mathrm{e}^{-\mathrm{iq} x^{1}+\mathrm{iq} t} \\
x^{1}>t & : & + & + & - & - \\
-t<x^{1}<t & : & - & + & - & + \\
x^{1}<-t & : & - & - & + & +
\end{array}
$$

Upon deformation, singularities of the function $U\left(p^{1}, \mathrm{q}\right)$ are crossed. These are simple poles at $\mathrm{q}= \pm p^{1}$. Because of the imaginary shift $\pm \mathrm{i} \mathbf{0}$ in (71), they are crossed only if $p^{1}<0$ when deforming the q-contour towards the positive imaginary direction, and only if $p^{1}>0$ when deforming the q-contour towards the negative imaginary direction. Remembering that $q>0$, only one of the two poles is crossed in any case. In both cases of the sign in $q= \pm p^{1}$, at the position of these poles we have $E_{q, \tilde{p}}=E_{p}$. Hence, at the position of these poles, $a_{\mathrm{q}, p}^{+}=1$ and $a_{\mathrm{q}, p}^{-}=0$. This means that in order to evaluate the associated residues, it is sufficient to consider only the terms with large-q factors $\mathrm{e}^{\mathrm{iq} x^{1}-\mathrm{iq} t}$ and $\mathrm{e}^{-\mathrm{iq} x^{1}-\mathrm{iq} t}$.

In the case $x^{1}>t$, we shift in the directions $(+)$ and $(-)$ for the terms containing $\mathrm{e}^{\mathrm{i} q x^{1}-\mathrm{iq} t}$ and $\mathrm{e}^{-\mathrm{iq} x^{1}-\mathrm{iq} t}$, respectively. In the first shift, we cross the pole of $U\left(p^{1}, \mathrm{q}\right)$ at $\mathrm{q}=-p^{1}$, and in the second, we cross the pole of $U\left(p^{1},-\mathrm{q}\right)$ at $-\mathrm{q}=p^{1}$, in both cases only if $p^{1}<0$. One can do a similar analysis for $x^{1}<-t$. The result, including the hermitian conjugate, is

$$
\phi(x, t) \stackrel{\left|x^{1}\right|>t}{=} \int D p\left(B_{p} \mathrm{e}^{\mathrm{i} \tilde{p} \cdot \tilde{x}-\mathrm{i} E_{p} t}+B_{p}^{\dagger} \mathrm{e}^{-\mathrm{i} \tilde{p} \cdot \tilde{x}+\mathrm{i} E_{p} t}\right) 2 \cos \left(p_{1} x_{1}\right) \Theta\left(-p_{1} x_{1}\right)+\text { integral contribution }
$$


where the integral contribution, calculated below, comes from the shifted integral itself. The terms explicitly written on right-hand side are, according to the first equation of (35), equal to $\mathrm{e}^{\mathrm{i} H_{0} t} \phi(x) \mathrm{e}^{-\mathrm{i} H_{0} t}$ where

$$
H_{0}=H_{\mathrm{L}}+H_{\mathrm{R}}
$$

In the case $-t<x^{1}<t$, we shift in the direction $(-)$ for both terms containing $\mathrm{e}^{\mathrm{iq} x^{1}-\mathrm{iq} t}$ and $\mathrm{e}^{-\mathrm{iq} x^{1}-\mathrm{iq} t}$. In the first shift we cross the pole of $U\left(p^{1}, \mathrm{q}\right)$ at $\mathrm{q}=p^{1}$ if $p^{1}>0$, and in the second, we cross the pole of $U\left(p^{1},-\mathrm{q}\right)$ at $-\mathrm{q}=p^{1}$ if $p^{1}<0$. Together, we obtain

$$
\phi(x, t) \stackrel{\left|x^{1}\right|<t}{=} \int D p\left(B_{p} \mathrm{e}^{-\mathrm{i} E_{p} t+\mathrm{i} p \cdot x}+B_{p}^{\dagger} \mathrm{e}^{\mathrm{i} E_{p} t-\mathrm{i} p \cdot x}\right)+\text { integral contribution }
$$

where again the integral contribution coems from the shifted integral itself. The explicit terms on the right-hand side have the structure of (34), except for the replacement $A_{p}, A_{p}^{\dagger} \mapsto B_{p}, B_{p}^{\dagger}$. That is, recalling the scattering isomorphism $S$ (39), we may write them as $S(\phi(x, t))$.

Finally, we calculate the integral contributions, from the shifted q-integral. After performing the deformations the q-integral (72) runs, for the various terms, between 0 and $\pm \mathrm{i} \infty$. In order to assess the result, we have to separate the region $|\mathrm{q}|>E_{\tilde{p}}:=\sqrt{|\tilde{p}|^{2}+m^{2}}$ from the region $|\mathrm{q}|<E_{\tilde{p}}$, because there are branch points at $\mathrm{q}= \pm \mathrm{i} E_{\tilde{p}}$ with branch cuts going towards $\pm \mathrm{i} \infty$. After deformation, we change the $\mathrm{q}$ variable to $\mathrm{q}= \pm \mathrm{i} w$ and we are left with integrals $\int_{0}^{ \pm \mathrm{i} \infty} \mathrm{d} q=$ $\pm \mathrm{i} \int_{0}^{\infty} \mathrm{d} w$. In the case $w>E_{\tilde{p}}$, we may use $E_{ \pm \mathrm{i} w, \tilde{p}}= \pm \mathrm{i} \sqrt{w^{2}-|\tilde{p}|^{2}-m^{2}}$, which implies $a_{\mathrm{i} w, p}^{ \pm}=$ $a_{-\mathrm{i} w, p}^{\mp}$. On the other hand, in the case $w<E_{\tilde{p}}$, we have $E_{ \pm \mathrm{i} w, \tilde{p}}=\sqrt{|\tilde{p}|^{2}+m^{2}-w^{2}}$ and $a_{\mathrm{i} w, p}^{ \pm}=a_{-\mathrm{i} w, p}^{ \pm}$.

Putting these rules together, in the case $\left|x^{1}\right|>t$, a straightforward calculation shows that both in the regions $w>E_{\tilde{p}}$ and $0<w<E_{\tilde{p}}$, the integrand in (72), arising after the deformation ,,,++-- or,,,--++ (as per (73)), is exactly zero. That is, the integral contribution is exactly zero in (74).

In the case $\left|x^{1}\right|<t$, in the region $w>E_{\tilde{p}}$ the integrand (72) also gives zero after the deformation,,,-+-+ , but it is non-zero in the region $0<w<E_{\tilde{p}}$. This contribution is (with $\left.p=\left(p^{1}, \tilde{p}\right)\right)$

$$
G\left(x^{1}, t ; p\right):=\frac{\mathrm{i} \operatorname{sign}\left(p^{1}\right)}{\pi} \int_{0}^{E_{\tilde{p}}} \mathrm{~d} w \frac{w \sinh \left(w x^{1}\right)}{w^{2}+\left(p^{1}\right)^{2}}\left(\left(1+\frac{E_{p}}{\mathcal{E}_{w, \tilde{p}}}\right) \mathrm{e}^{-\mathrm{i} \mathcal{E}_{w, \tilde{p}} t}-\left(1-\frac{E_{p}}{\mathcal{E}_{w, \tilde{p}}}\right) \mathrm{e}^{\mathrm{i} \mathcal{E}_{w, \tilde{p}} t}\right)
$$

where

$$
\mathcal{E}_{w, \tilde{p}}=\sqrt{|\tilde{p}|^{2}+m^{2}-w^{2}} .
$$

Changing variable to $u=\mathcal{E}_{w, \tilde{p}}$, this simplifies to (43)

Recall that the above calculation was performed with initial (before-quench) free condition on $\phi(x)$ at $x^{1}=0$. A similar calculation may be done for initial fixed condition at $x^{1}=0$. The factor $\cos \left(p^{1} x^{1}\right)$ is replaced by $i \sin \left(p^{1} x^{1}\right)$ in (35). The result is as above, but with the correction factor (44) instead of that given by (43). Technically, this may be obtained by observing that one can go from fixed to free condition by formally applying the operator $\left(\mathrm{i} p^{1}\right)^{-1} \partial / \partial x^{1}$ inside the $p$-integral (see the expression (35), with $\cos \left(p^{1} x^{1}\right)$ is replaced by $i \sin \left(p^{1} x^{1}\right)$ ); the sine function guarantees that the delta-function coming from the factor $\Theta\left(-p^{1} x^{1}\right)$ does not contribute.

Finally, let us discuss the convergence properties of expressions obtained from (41). Consider evaluating the average of a local operator $\mathcal{O}(x)$ at $x$, formed by normal-ordered products of $\phi(x)$, 
$\pi(x)$ and their derivatives. One uses (41) and (37) along with Wick's theorem. Integrating over momenta, we see that the integrands will be suppressed by factors $e^{-|p| \beta_{\mathrm{L}, \mathrm{R}}}$ at large momenta for every Wick contraction, due to the denominator in the second equation of (37) (the one that is relevant for normal-ordered operators). Contractions that involve two $G$-corrections have two factors $G\left(x^{1}, t ; p\right)$, integrated over $p$. Due to the sinh (resp. cosh) factor in the integrand in (43) (resp. (44)), the large- $|\tilde{p}|$ behaviour of the integrand along the integration path has a factor $e^{2|\tilde{p}|\left|x^{1}\right|}$ (for $u$ away from the integration limits). Hence, this shows convergence of the resulting integral only for $\left|x^{1}\right|<2 \min \left(\beta_{\mathrm{L}, \mathrm{R}}\right)$. For values of $x^{1}$ beyond this region, one may modify the integral representation to show convergence beyond this region. A convenient way is to shift the $u$ contour towards the positive imaginary direction. The result, which we report here for the initially free case, is:

$$
\begin{gathered}
G\left(x^{1}, t ; p\right) \\
=-\frac{\operatorname{sign}\left(p^{1}\right)}{\pi} \int_{0}^{\infty} \mathrm{d} v e^{-v t}\left(\frac{\sinh \left(\sqrt{v^{2}+2 i v E_{\tilde{p}}} x^{1}\right)}{E_{p}+\mathrm{i} v-E_{\tilde{p}}} \mathrm{e}^{-\mathrm{i} E_{\tilde{p}} t}-\frac{\sinh \left(\sqrt{v^{2}-2 \mathrm{i} v E_{\tilde{p}}} x^{1}\right)}{E_{p}+\mathrm{i} v+E_{\tilde{p}}} \mathrm{e}^{\mathrm{i} E_{\tilde{p}} t}\right) \\
\left.\quad \text { (initially free at } x^{1}=0\right)
\end{gathered}
$$

The integrand now diverges much more slowly at large $|\tilde{p}|$, which guarantees convergence. We use such representations in the next subsection in order to perform the large-time asymptotic analysis of the energy current average.

\section{Asymptotic time evolution of the energy current}

We consider the large-time asymptotic of the average $\left\langle T^{01}(t, 0)\right\rangle$ with fixed boundary conditions at $d=1$, evaluated using (41) and (12).

Performing the trace and then the integration over the angles in the transverse direction, we find, in generic dimensions $d$,

$$
\begin{aligned}
\left\langle T^{01}(x, t)\right\rangle \stackrel{\left|x^{1}\right|<t}{=} & \left\langle T^{01}\right\rangle_{\mathrm{s}}+\frac{1}{2^{d} \pi^{\frac{d+1}{2}} \Gamma\left(\frac{d-1}{2}\right)} \int_{-\infty}^{\infty} \mathrm{d} p^{1} \int_{0}^{\infty} \mathrm{d} \tilde{p} \frac{\tilde{p}^{d-2}}{E_{p}\left(\mathrm{e}^{W(p)}-1\right)} \times \\
& \times\left[2 p^{1} \operatorname{Im}\left(\mathrm{e}^{\mathrm{i} p^{1} x^{1}-\mathrm{i} E_{p} t} \partial_{0} \bar{G}\right)+2 E_{p} \operatorname{Im}\left(\mathrm{e}^{-\mathrm{i} p^{1} x^{1}+\mathrm{i} E_{p} t} \partial_{1} G\right)-2 \operatorname{Re}\left(\partial_{0} G \partial_{1} \bar{G}\right)\right] .
\end{aligned}
$$

For simplicity, we will restrict ourselves to $d=1$. For $d=1$ the derivation, without the angular integration in the transverse direction (and omitting the upper-1 space index), gives

$$
\begin{aligned}
\left\langle T^{01}(x, t)\right\rangle \stackrel{|x|<t, d=1}{=} & \left\langle T^{01}\right\rangle_{\mathrm{s}}+\int_{-\infty}^{\infty} \frac{\mathrm{d} p}{4 \pi E_{p}\left(\mathrm{e}^{W(p)}-1\right)} \times \\
& \times\left[2 p \operatorname{Im}\left(\mathrm{e}^{\mathrm{i} p x-\mathrm{i} E_{p} t} \partial_{0} \bar{G}\right)+2 E_{p} \operatorname{Im}\left(\mathrm{e}^{-\mathrm{i} p x+\mathrm{i} E_{p} t} \partial_{1} G\right)-2 \operatorname{Re}\left(\partial_{0} G \partial_{1} \bar{G}\right)\right] .
\end{aligned}
$$

We use fixed initial boundary conditions, for which the function $G$ is

$$
G\left(x^{1}, t ; p\right)=-\frac{\left|p^{1}\right|}{\pi} \int_{-E_{\tilde{p}}}^{E_{\tilde{p}}} \mathrm{~d} u \frac{\cosh \left(\mathcal{E}_{u, \tilde{p}} x^{1}\right)}{\left(E_{p}+u\right) \mathcal{E}_{u, \tilde{p}}} \mathrm{e}^{\mathrm{i} u t} .
$$

Again, for $d=1$ this specializes to

$$
G(x, t ; p)=-\frac{|p|}{\pi} \int_{-m}^{m} \mathrm{~d} u \frac{\cosh \left(\sqrt{m^{2}-u^{2}} x\right)}{\left(E_{p}+u\right) \sqrt{m^{2}-u^{2}}} \mathrm{e}^{\mathrm{i} u t} .
$$


We have

$$
\partial_{1} G=-\frac{|p|}{\pi} \int_{-m}^{m} \mathrm{~d} u \frac{\sinh \left(\sqrt{m^{2}-u^{2}} x\right)}{\left(E_{p}+u\right)} \mathrm{e}^{\mathrm{i} u t} .
$$

Specializing at $x=0$, this is zero. Hence, in the expression for the current (81), only the first term remains and we have

$$
\left\langle T^{01}(t, 0)\right\rangle=\left\langle T^{01}\right\rangle_{\mathrm{s}}+\int_{-\infty}^{\infty} \frac{\mathrm{d} p}{4 \pi E_{p}\left(\mathrm{e}^{W(p)}-1\right)} 2 p \operatorname{Im}\left(\mathrm{e}^{-\mathrm{i} E_{p} t} \partial_{0} \bar{G}(0, t ; p)\right)
$$

where

$$
G(0, t ; p)=-\frac{|p|}{\pi} \int_{-m}^{m} \mathrm{~d} u \frac{\mathrm{e}^{\mathrm{i} u t}}{\left(E_{p}+u\right) \sqrt{m^{2}-u^{2}}}
$$

We are interested in the correction

$$
\delta\left(\beta_{\mathrm{L}}, \beta_{\mathrm{R}}\right)=\int_{-\infty}^{\infty} \frac{\mathrm{d} p}{4 \pi E_{p}\left(\mathrm{e}^{W(p)}-1\right)} 2 p \operatorname{Im}\left(\mathrm{e}^{-\mathrm{i} E_{p} t} \partial_{0} \bar{G}(0, t ; p)\right) .
$$

We now recast (86) in a form where the asymptotic analysis can be made accurately. We deform the contour towards the positive imaginary direction, where it vanishes at positive imaginary infinity. There are two contributions, one on the line with real part $-m$ the other with real part $m$. For the first we change variable to $u=-m+\mathrm{i} v$ and for the other, $u=m+\mathrm{i} v$, with $v$ from 0 to $\infty$ in the first instance, and from $\infty$ to 0 in the second. This gives

$$
G(0, t ; p)=-\frac{\mathrm{i}|p|}{\pi} \int_{0}^{\infty} \mathrm{d} v \mathrm{e}^{-v t}\left(\frac{\mathrm{e}^{-\mathrm{i} m t}}{\left(E_{p}-m+\mathrm{i} v\right) \sqrt{v^{2}+2 \mathrm{i} m v}}-\frac{\mathrm{e}^{\mathrm{i} m t}}{\left(E_{p}+m+\mathrm{i} v\right) \sqrt{v^{2}-2 \mathrm{i} m v}}\right)
$$

and

$$
\begin{aligned}
\partial_{0} \bar{G}(0, t ; p) & =\frac{i|p|}{\pi} \int_{0}^{\infty} \mathrm{d} v \mathrm{e}^{-v t}\left(\frac{(\mathrm{i} m-v) \mathrm{e}^{\mathrm{i} m t}}{\left(E_{p}-m-\mathrm{i} v\right) \sqrt{v^{2}-2 \mathrm{i} m v}}+\frac{(\mathrm{i} m+v) \mathrm{e}^{-\mathrm{i} m t}}{\left(E_{p}+m-\mathrm{i} v\right) \sqrt{v^{2}+2 \mathrm{i} m v}}\right) \\
& =g_{+}+g_{-}
\end{aligned}
$$

where

$$
g_{ \pm}=\frac{\mathrm{i}|p|}{\pi} \int_{0}^{\infty} \mathrm{d} v \mathrm{e}^{-v t} \frac{(\mathrm{i} m \mp v) \mathrm{e}^{ \pm \mathrm{i} m t}}{\left(E_{p} \mp m-\mathrm{i} v\right) \sqrt{v^{2} \mp 2 \mathrm{i} m v}} .
$$

We consider the integral

$$
I(\beta, t)=\int_{0}^{\infty} \frac{\mathrm{d} p p \mathrm{e}^{-\mathrm{i} E_{p} t} \partial_{0} \bar{G}(0, t ; p)}{E_{p}\left(\mathrm{e}^{\beta E_{p}}-1\right)}=I_{+}+I_{-}
$$

where

$$
I_{ \pm}=\int_{0}^{\infty} \frac{\mathrm{d} p p \mathrm{e}^{-\mathrm{i} E_{p} t} g_{ \pm}}{E_{p}\left(\mathrm{e}^{\beta E_{p}}-1\right)} .
$$

The correction to the stationary value is given by

$$
\delta\left(\beta_{\mathrm{L}}, \beta_{\mathrm{R}}\right)=\frac{1}{2 \pi} \operatorname{Im}\left(I\left(\beta_{l}, t\right)-I\left(\beta_{r}, t\right)\right) .
$$


Changing variables we have

$$
I_{ \pm}=\frac{\mathrm{i}}{\pi} \int_{m}^{\infty} \mathrm{d} E \frac{\mathrm{e}^{-\mathrm{i}(E \mp m) t}}{\mathrm{e}^{\beta E}-1} \int_{0}^{\infty} \mathrm{d} v \frac{\mathrm{e}^{-v t}(\mathrm{i} m \mp v) \sqrt{E^{2}-m^{2}}}{(E \mp m-\mathrm{i} v) \sqrt{v^{2} \mp 2 \mathrm{i} m v}} .
$$

Let us concentrate on $I_{+}$. We find

$$
\begin{aligned}
I_{+} & =\frac{\mathrm{i}}{\pi} \int_{m}^{\infty} \mathrm{d} E \frac{\mathrm{e}^{-\mathrm{i}(E-m) t}}{\mathrm{e}^{\beta E}-1} \int_{0}^{\infty} \mathrm{d} v \frac{\mathrm{e}^{-v t}(\mathrm{i} m-v) \sqrt{E^{2}-m^{2}}}{(E-m-\mathrm{i} v) \sqrt{v^{2}-2 \mathrm{i} m v}} \\
& =\frac{\mathrm{i}}{\pi} \int_{0}^{\infty} \mathrm{d} E \frac{\mathrm{e}^{-\mathrm{i} E t}}{\mathrm{e}^{\beta(E+m)}-1} \int_{0}^{\infty} \mathrm{d} v \frac{\mathrm{e}^{-v t}(\mathrm{i} m-v) \sqrt{E} \sqrt{E+2 m}}{(E-\mathrm{i} v) \sqrt{v^{2}-2 \mathrm{i} m v}} .
\end{aligned}
$$

In terms of the variable $E$, there are singularities at $0,-2 m,-m+2 \pi \mathrm{i} n / \beta(n \in \mathbb{Z})$ and $i v$. Hence there are no singularities in the lower-right quadrant, and we can deform the contour towards the negative imaginary direction. With the replacement $E \mapsto-i E$, this gives

$$
I_{+}=\frac{\mathrm{i}(-\mathrm{i})^{\frac{1}{2}}}{\pi} \int_{0}^{\infty} \mathrm{d} E \frac{\mathrm{e}^{-E t}}{\mathrm{e}^{\beta(m-\mathrm{i} E)}-1} \int_{0}^{\infty} \mathrm{d} v \frac{\mathrm{e}^{-v t}(\mathrm{i} m-v) \sqrt{E} \sqrt{2 m-\mathrm{i} E}}{(E+v) \sqrt{v^{2}-2 \mathrm{i} m v}} .
$$

Since we have real exponentials $\mathrm{e}^{-E t}$ and $\mathrm{e}^{-v t}$, the asymptotic large- $t$ expansion is obtained by expanding the integrand around $E=0$ and $v=0$. We do that to first order. We have

$$
\begin{aligned}
\sqrt{2 m-\mathrm{i} E} & \approx \sqrt{2 m}\left(1-\frac{\mathrm{i} E}{4 m}\right) \\
\frac{1}{\mathrm{e}^{\beta(m-\mathrm{i} E)-1}} & \approx \frac{1}{\mathrm{e}^{\beta m}(1-\mathrm{i} \beta E)-1} \\
& =\frac{1}{\left(\mathrm{e}^{\beta m}-1\right)\left(1-\frac{\mathrm{i} \beta E}{1-\mathrm{e}^{-\beta m}}\right)} \\
& \approx \frac{1}{\mathrm{e}^{\beta m}-1}\left(1+\frac{\mathrm{i} \beta E}{1-\mathrm{e}^{-\beta m}}\right) \\
\frac{1}{\sqrt{v^{2}-2 \mathrm{i} m v}} & \approx \frac{1}{\sqrt{-2 \mathrm{i} m} \sqrt{v}}\left(1+\frac{v}{4 \mathrm{i} m}\right) \\
\mathrm{i} m-v & =i m\left(1-\frac{v}{\mathrm{i} m}\right) .
\end{aligned}
$$

This gives

$$
I_{+} \approx-\frac{m}{\pi} \frac{1}{\mathrm{e}^{\beta m}-1} \int_{0}^{\infty} \mathrm{d} E \mathrm{~d} v \frac{e^{-(E+v) t}}{E+v} \sqrt{\frac{E}{v}}\left(1-\frac{\mathrm{i} E}{4 m}+\frac{\mathrm{i} \beta E}{1-\mathrm{e}^{-\beta m}}+\frac{v}{4 \mathrm{i} m}-\frac{v}{\mathrm{i} m}\right) .
$$


We now evaluate the following integrals:

$$
\begin{aligned}
\int_{0}^{\infty} \mathrm{d} E \mathrm{~d} v \frac{\mathrm{e}^{-(E+v) t}}{E+v} \sqrt{\frac{E}{v}} E^{j} v^{k} & =\int_{t}^{\infty} \mathrm{d} s \int_{0}^{\infty} \mathrm{d} E \mathrm{~d} v \mathrm{e}^{-(E+v) s} \sqrt{\frac{E}{v}} E^{j} v^{k} \\
& =\int_{t}^{\infty} \mathrm{d} s s^{-2-j-k} \Gamma(j+3 / 2) \Gamma(k+1 / 2) \\
& =\frac{t^{-1-j-k}}{1+j+k} \Gamma(j+3 / 2) \Gamma(k+1 / 2) \\
& =\left\{\begin{array}{cl}
\frac{\pi}{2 t} & (j=k=0) \\
\frac{3 \pi}{8 t^{2}} & (j=1, k=0) \\
\frac{\pi}{8 t^{2}} & (j=0, k=1) .
\end{array}\right.
\end{aligned}
$$

Putting these together,

$$
\begin{aligned}
I_{+} & \approx-\frac{m}{2 t} \frac{1}{\mathrm{e}^{\beta m}-1}\left(1-\frac{3 \mathrm{i}}{16 m t}+\frac{3}{4 t} \frac{\mathrm{i} \beta}{1-\mathrm{e}^{-\beta m}}-\frac{3}{16 \mathrm{i} m t}\right) \\
& =-\frac{m}{2 t} \frac{1}{\mathrm{e}^{\beta m}-1}\left(1+\frac{3}{4 t} \frac{\mathrm{i} \beta}{1-\mathrm{e}^{-\beta m}}\right) .
\end{aligned}
$$

Hence this contribution is

$$
\frac{1}{2 \pi} \operatorname{Im}\left(I_{+}\right)=-\frac{3 m \beta}{64 \pi \sinh ^{2}(\beta m / 2)} t^{-2}+O\left(t^{-3}\right) .
$$

Let us next concentrate on $I_{-}$. We have, following similar lines,

$$
\begin{aligned}
I_{-} & =\frac{\mathrm{i}}{\pi} \int_{m}^{\infty} \mathrm{d} E \frac{\mathrm{e}^{-\mathrm{i}(E+m) t}}{\mathrm{e}^{\beta E}-1} \int_{0}^{\infty} \mathrm{d} v \frac{\mathrm{e}^{-v t}(\mathrm{i} m+v) \sqrt{E^{2}-m^{2}}}{(E+m-\mathrm{i} v) \sqrt{v^{2}+2 \mathrm{i} m v}} \\
& =\frac{\mathrm{i}}{\pi} \int_{0}^{\infty} \mathrm{d} E \frac{\mathrm{e}^{-\mathrm{i}(E+2 m) t}}{\mathrm{e}^{\beta(E+m)}-1} \int_{0}^{\infty} \mathrm{d} v \frac{\mathrm{e}^{-v t}(\mathrm{i} m+v) \sqrt{E} \sqrt{E+2 m}}{(E+2 m-\mathrm{i} v) \sqrt{v^{2}+2 \mathrm{i} m v}} \\
& =\frac{\mathrm{i}(-\mathrm{i})^{\frac{1}{2}} \mathrm{e}^{-2 \mathrm{i} m t}}{\pi} \int_{0}^{\infty} \mathrm{d} E \frac{\mathrm{e}^{-E t}}{\mathrm{e}^{\beta(m-\mathrm{i} E)}-1} \int_{0}^{\infty} \mathrm{d} v \frac{\mathrm{e}^{-v t}(\mathrm{i} m+v) \sqrt{E} \sqrt{2 m-\mathrm{i} E}}{(E+v+2 \mathrm{i} m) \sqrt{v^{2}+2 \mathrm{i} m v}} .
\end{aligned}
$$

Now it is sufficient to expand to zeroth order, obtaining

$$
\begin{aligned}
I_{-} & \approx \frac{\mathrm{i}(-\mathrm{i})^{\frac{1}{2}} \mathrm{e}^{-2 \mathrm{i} m t}}{2 \pi\left(\mathrm{e}^{\beta m}-1\right)} \int_{0}^{\infty} \mathrm{d} E \mathrm{e}^{-E t} \int_{0}^{\infty} \mathrm{d} v \frac{\mathrm{e}^{-v t} \sqrt{E} \sqrt{2 m}}{\sqrt{2 \mathrm{i} m v}} \\
& =\frac{\mathrm{e}^{-2 \mathrm{i} m t}}{2 \pi\left(\mathrm{e}^{\beta m}-1\right)} \int_{0}^{\infty} \mathrm{d} E \mathrm{~d} v \mathrm{e}^{-(E+v) t} \sqrt{\frac{E}{v}} \\
& =\frac{\mathrm{e}^{-2 \mathrm{i} m t}}{2 \pi\left(\mathrm{e}^{\beta m}-1\right)} t^{-2} \Gamma(3 / 2) \Gamma(1 / 2) \\
& =\frac{\mathrm{e}^{-2 \mathrm{i} m t}}{4\left(e^{\beta m}-1\right)} t^{-2}
\end{aligned}
$$

Hence the contribution is

$$
\frac{1}{2 \pi} \operatorname{Im}\left(I_{-}\right)=-\frac{\sin (2 m t)}{8 \pi\left(\mathrm{e}^{\beta m}-1\right)} t^{-2}+O\left(t^{-3}\right) .
$$


Putting the above results together, we find $\delta\left(\beta_{\mathrm{L}}, \beta_{\mathrm{R}}\right)=\delta\left(\beta_{\mathrm{L}}\right)-\delta\left(\beta_{\mathrm{R}}\right)$ where $\delta(\beta)$ is given in (54). We note that the correction has an oscillatory part, and that it is always negative (for all $\beta_{\mathrm{L}, \mathrm{R}} m>0$ and $\beta_{\mathrm{L}}<\beta_{\mathrm{R}}$, with $\delta(\infty)=0$.

\section{E Numerical simulations}

An independent check of our analytical calculations are provided by a first principles numerical simulation of the evolution of the free field theory. We will solve this problem for the case $d=1$, for a massive boson. The higher dimensional cases may be solved by this method as well, as the transverse momenta act as an effective mass $m_{\text {eff }}^{2} \equiv \sqrt{\tilde{p}^{2}+m^{2}}$, from the point of view of any observable dependent only on $x$ and $t$. The simplest way to regulate this theory numerically is to place the system in a large, but finite box, of length $\mathcal{L}$. Let us denote $0<X<\mathcal{L}$ with the spatial coordinate of the box - in the continuum theory, $x=X-\mathcal{L} / 2$. Choosing boundary conditions $\phi(X=0)=\phi(X=\mathcal{L})=0$, we may write the (classical) field

$$
\phi(x, t)=\sum_{n=1}^{\infty} a_{n}(t) \sin \left(\frac{n \pi X}{\mathcal{L}}\right),
$$

As in the main text, it will suffice to compute solutions of the classical equations of motion, to solve the full quantum evolution for a free theory. In particular, we need to time-evolve the finite-box equivalent of the half-modes $\cos (p \cdot x) \Theta\left(-p^{1} x\right)$. These modes are characterized by an integer $m$, which we take to be odd if we wish for, in the continuum theory, $\phi$ to satisfy free boundary conditions at the interface; $m$ is even if the boundary conditions are fixed. Without loss of generality, let us consider modes propagating from left to right. Since this is a free theory, the appropriate Green's functions are given by

$$
\mathcal{G}_{l}(t=0)=\sum_{n=1}^{\infty} \mathcal{A}_{l, n}(t) \sin \frac{n \pi X}{\mathcal{L}}
$$

where

$$
\mathcal{A}_{l, n}(t) \equiv \begin{cases}\frac{\sqrt{2}}{\pi}\left(\frac{\sin ((l-n) \pi / 2)}{l-n}-\frac{\sin ((l+n) \pi / 2)}{l+n}\right)\left(\cos \left(E_{n} t\right)-\mathrm{i} \frac{E_{l}}{E_{n}} \sin \left(E_{n} t\right)\right) & l \neq n \\ \frac{\mathrm{e}^{-\mathrm{i} E_{l} t}}{\sqrt{2}} & l=n\end{cases}
$$

with

$$
E_{n} \equiv \sqrt{m^{2}+\frac{n^{2} \pi^{2}}{\mathcal{L}^{2}}} .
$$

These Green's functions are straightforwardly used to evaluate correlation functions. For example, let us consider the case where $d=1, T_{\mathrm{L}}=T$, and $T_{\mathrm{R}}=0$. Then

$$
\left\langle T^{01}(X, t)\right\rangle=\sum_{l} \frac{2}{\mathcal{L}} \frac{1}{\mathrm{e}^{E_{l} / T}-1}\left[-\operatorname{Re}\left(\partial_{x} \mathcal{G}_{l} \partial_{t} \mathcal{G}_{l}\right)\right]
$$

where the sum over $m$ is only over odd positive integers (free boundary conditions) or even positive integers (fixed boundary conditions). 
In practice, we must truncate the sum over integers to a finite number. We have typically chosen $T, m \sim \mathcal{O}(1), \mathcal{L}=200$ and truncated the sum to include $m \leq 800$, which is adequate to find convergence.

\section{F Correlation functions in the steady state}

Here we compute the algebraic decay of the $\langle\phi(x) \pi(0)\rangle$ correlation function in $d=1$ massive Klein-Gordon theory at late times after the local quench. In order to show (33), we use the trace relations (11) along with (4). We find, in $d=1$,

$$
\begin{aligned}
\langle\phi(x) \pi(0)\rangle_{\mathrm{s}} & =i \int_{-\infty}^{\infty} \frac{\mathrm{d} p}{4 \pi}\left(\frac{\mathrm{e}^{\mathrm{i} p x^{1}}}{1-\mathrm{e}^{-W(p)}}-\frac{\mathrm{e}^{-\mathrm{i} p x^{1}}}{\mathrm{e}^{W(p)}-1}\right) \\
& =i \int_{0}^{\infty} \frac{d p}{4 \pi}\left(\frac{\mathrm{e}^{\mathrm{i} p x^{1}}}{1-\mathrm{e}^{-\beta_{\mathrm{L}} E_{p}}}-\frac{\mathrm{e}^{-\mathrm{i} p x^{1}}}{\mathrm{e}^{\beta_{\mathrm{L}} E_{p}}-1}\right)+\left(\left(\beta_{\mathrm{L}}, x^{1}\right) \mapsto\left(\beta_{\mathrm{R}},-x^{1}\right)\right) .
\end{aligned}
$$

The asymptotics of the integral can be evaluated by contour deformation. Let us assume $x^{1}>0$. Then:

$$
\begin{aligned}
\mathrm{i} & \int_{0}^{\infty} \frac{\mathrm{d} p}{4 \pi}\left(\frac{\mathrm{e}^{\mathrm{i} p x^{1}}}{1-\mathrm{e}^{-\beta_{\mathrm{L}} E_{p}}}-\frac{\mathrm{e}^{-\mathrm{i} p x^{1}}}{\mathrm{e}^{\beta_{\mathrm{L}} E_{p}}-1}\right) \\
& =-\int_{0}^{m} \frac{\mathrm{d} \ell}{4 \pi} e^{-\ell x^{1}}\left(\frac{1}{1-\mathrm{e}^{-\beta_{\mathrm{L}} \sqrt{m^{2}-\ell^{2}}}}+\frac{1}{\mathrm{e}^{\beta_{\mathrm{L}} \sqrt{m^{2}-\ell^{2}}}-1}\right)+O\left(\mathrm{e}^{-m x^{1}}\right) \\
& \sim-\int_{0}^{\infty} \frac{\mathrm{d} \ell}{4 \pi} \mathrm{e}^{-\ell x^{1}} \operatorname{coth} \frac{\beta_{\mathrm{L}} m}{2} \\
& =-\frac{1}{4 \pi x^{1}} \operatorname{coth} \frac{\beta_{\mathrm{L}} m}{2}
\end{aligned}
$$

Hence we find (33). A similar analysis for $\langle\phi(x) \phi(0)\rangle_{\mathrm{s}}$ gives an exponential decay instead of an algebraic decay. Indeed, we have

$$
\langle\phi(x) \phi(0)\rangle_{\mathrm{s}}=\int_{0}^{\infty} \frac{\mathrm{d} p}{4 \pi E_{p}}\left(\frac{\mathrm{e}^{\mathrm{i} p x^{1}}}{1-\mathrm{e}^{-\beta_{\mathrm{L}} E_{p}}}+\frac{\mathrm{e}^{-\mathrm{i} p x^{1}}}{\mathrm{e}^{\beta_{\mathrm{L}} E_{p}}-1}\right)+\left(\left(\beta_{\mathrm{L}}, x^{1}\right) \mapsto\left(\beta_{\mathrm{R}},-x^{1}\right)\right)
$$

and

$$
\begin{aligned}
& \int_{0}^{\infty} \frac{\mathrm{d} p}{4 \pi E_{p}}\left(\frac{\mathrm{e}^{\mathrm{i} p x^{1}}}{1-\mathrm{e}^{-\beta_{\mathrm{L}} E_{p}}}+\frac{\mathrm{e}^{-\mathrm{i} p x^{1}}}{\mathrm{e}^{\beta_{\mathrm{L}} E_{p}}-1}\right) \\
& =i \int_{0}^{m} \frac{\mathrm{d} \ell}{4 \pi \sqrt{m^{2}-\ell^{2}}} \mathrm{e}^{-\ell x^{1}}\left(\frac{1}{1-e^{-\beta_{\mathrm{L}} \sqrt{m^{2}-\ell^{2}}}}-\frac{1}{e^{\beta_{\mathrm{L}} \sqrt{m^{2}-\ell^{2}}-1}}\right)+O\left(\mathrm{e}^{-m x^{1}}\right) \\
& =i \int_{0}^{m} \frac{\mathrm{d} \ell}{4 \pi \sqrt{m^{2}-\ell^{2}}} \mathrm{e}^{-\ell x^{1}}+O\left(\mathrm{e}^{-m x^{1}}\right) \\
& =\sum_{k=0}^{\infty} \frac{a_{k}}{x^{2 k+1}}+O\left(\mathrm{e}^{-m x^{1}}\right)
\end{aligned}
$$

where the constants $a_{k}$ are obtained from expanding the integrand in $\ell^{2}$ in the penultimate line. Putting this into (115), the algebraic part cancels out, thus showing exponential decay of the correlation function. 


\section{G Dimensional reduction}

Dimensional reduction through integration over "perpendicular" coordinates is a phenomenon that has been observed in various situations, perhaps the most prominent being the reduction of electronic leads from three to one dimension in the context of impurity models. Here we present the general theory for the Klein-Gordon model.

Let $1 \leq D<d$ and consider the following fields, which are integration over a $(d-D)$ dimensional subspace of the fundamental Klein-Gordon fields:

$$
\Phi(\mathrm{x}):=\frac{1}{L^{\frac{D-d}{2}}} \int_{-\frac{L}{2}}^{\frac{L}{2}} \mathrm{~d}^{d-D} \tilde{x} \phi(x), \quad \Pi(\mathrm{x}):=\frac{1}{L^{\frac{D-d}{2}}} \int_{-\frac{L}{2}}^{\frac{L}{2}} \mathrm{~d}^{d-D} \tilde{x} \pi(x)
$$

where $\mathrm{x}=\left(x^{1}, \ldots, x^{D}\right)$ and $\tilde{x}=\left(x^{D+1}, \ldots, x^{d}\right)$. Consider also their time-evolution $\Phi(t, \mathrm{x})=$ $\mathrm{e}^{\mathrm{i} H t} \Phi(\mathrm{x}) \mathrm{e}^{-\mathrm{i} H t}, \Pi(t, \mathrm{x})=\mathrm{e}^{\mathrm{i} H t} \Pi(\mathrm{x}) \mathrm{e}^{-\mathrm{i} H t}$. Using integration by parts and neglecting the boundary terms (which can be safely done in the limit $L \rightarrow \infty$ ), we verify the following:

$$
\dot{\Phi}(t, \mathrm{x}):=\Pi(\mathrm{x}), \quad \dot{\Pi}(t, \mathrm{x}) \stackrel{L \rightarrow \infty}{=}\left(\nabla_{\mathrm{x}}^{2}-m^{2}\right) \Phi(t, \mathrm{x}) .
$$

Further, from (2), we find the following equal-time commutation relations at large $L$ :

$$
[\Phi(\mathrm{x}), \Phi(\mathrm{y})]=[\Pi(\mathrm{x}), \Pi(\mathrm{y})]=0, \quad[\Phi(\mathrm{x}), \Pi(\mathrm{y})] \stackrel{L \rightarrow \infty}{=} \mathrm{i} \delta^{D}(\mathrm{x}-\mathrm{y}) .
$$

The vacuum $|\mathrm{vac}\rangle$ of the $d$-dimensional Klein-Gordon theory, defined in subsection 2.1 using mode operators, may be equivalently defined by the conditions

$$
\lim _{\tau \rightarrow-\infty} \phi(x,-\mathrm{i} \tau)|\mathrm{vac}\rangle=\lim _{\tau \rightarrow-\infty} \pi(x,-\mathrm{i} \tau)|\mathrm{vac}\rangle=0 .
$$

Hence we also have

$$
\lim _{\tau \rightarrow-\infty} \Phi(x,-\mathrm{i} \tau)|\mathrm{vac}\rangle=\lim _{\tau \rightarrow-\infty} \Pi(x,-\mathrm{i} \tau)|\mathrm{vac}\rangle=0 .
$$

Relations (117), (118) and (120) (for $L \rightarrow \infty$ ) define the $D$-dimensional Klein-Gordon theory for the canonical fields $\Phi(t, \mathrm{x})$ and $\Pi(t, \mathrm{x})$ and the vacuum $|\mathrm{vac}\rangle$. That is, the equations (116) for $L \rightarrow \infty$ can be seen as defining a homomorphism $\Omega: d$-dim KG $\rightarrow D$-dim KG from a $d$ dimensional to a $D$-dimensional Klein-Gordon theory. In particular, correlation functions of $\Phi(t, \mathrm{x})$ and $\Pi(t, \mathrm{x})$ in the vacuum, seen as integrals of correlation functions in the $d$-dimensional Klein-Gordon theory via (116), can be calculated using the $D$-dimensional Klein-Gordon theory.

Note that the facts that the time evolution generated by $H$ is the natural one in the $D$ dimensional theory and that it annihilates $|\mathrm{vac}\rangle$, along with (118), implies that, on the $D$ dimensional theory (the image of $\Omega$ ),

$$
\left.H\right|_{\operatorname{Im}(\Omega)}=\frac{1}{2} \int \mathrm{d}^{D} \mathrm{x}:\left(\Pi(\mathrm{x})^{2}+(\nabla \Phi(\mathrm{x}))^{2}\right): .
$$

Recall (6). The local equations of motion generated by $H_{\mathrm{L}, \mathrm{R}}$ take the same form on the fields $\Phi(t, \mathrm{x})$ and $\Pi(t, \mathrm{x})$ for any $D$, by similar arguments as those above. This implies that we also have

$$
\left.H_{\mathrm{L}, \mathrm{R}}\right|_{\operatorname{Im}(\Omega)}=\frac{1}{2} \int_{x_{1} \lessgtr 0} \mathrm{~d}^{D} \mathrm{x}:\left(\Pi(\mathrm{x})^{2}+(\nabla \Phi(\mathrm{x}))^{2}+m^{2} \Phi(\mathrm{x})^{2}\right): .
$$


Hence, the initial density matrix $\rho_{0}(7)$ has the same form for any $D$, in terms of the integrated fields. This means that, from the viewpoint of the integrated fields, the $d$-dimensional quench problem is equivalent to the $D$-dimensional quench problem for the Klein-Gordon theory. Hence in particular the steady-state density matrix, on $\operatorname{Im}(\Omega)$, also has the same form (9) in terms of the modes of the $D$-dimensional Klein-Gordon fields $\Phi(\mathrm{x})$ and $\Pi(\mathrm{x})$.

Naturally, the normal-ordering operation of the $d$-dimensional theory is mapped under $\Omega$ to the $D$-dimensional normal-ordering. However, one can consider a different set of operators involving products of fields, which does not lie in $\operatorname{Im}(\Omega)$. Consider the set of all fields of the form

$$
L^{\frac{(n-2)(D-d)}{2}} \int_{-\frac{L}{2}}^{\frac{L}{2}} \mathrm{~d}^{d-D} \tilde{x} \mathcal{O}_{1}\left(t_{1}, \mathrm{x}_{1}, \tilde{x}\right) \cdots \mathcal{O}_{n}\left(t_{n}, \mathrm{x}_{n}, \tilde{x}\right)
$$

for $\mathcal{O}_{i} \in\{\phi, \pi\}$ and $n$ a positive integer. This set of fields generates the same Lie algebra (under commutation) as does the set

$$
\mathcal{O}_{1}\left(t_{1}, \mathrm{x}_{1}\right) \cdots \mathcal{O}_{n}\left(t_{n}, \mathrm{x}_{n}\right)
$$

for $\mathcal{O}_{i} \in\{\Phi, \Pi\}$ and $n$ a positive integer, within the $D$-dimensional Klein-Gordon theory. With $n=1$, the operators do lie in $\operatorname{Im}(\Omega)$ and this is just a consequence of the fact that $\Omega$ is a homomorphism of Klein-Gordon theories. Considering commutators between $n=2$ and $n=1$ operators, and the fact that the normal-ordering of a $n=2$ operator only adds a term proportional to the identity operator, we find that equations (121) and (122) indeed agree with the above Lie algebra statement.

This technique of dimensional reduction can be applied straightforwardly to any free-field theory. We will discuss similar ideas in general QFT in a forthcoming work.

Finally, we note that applying the results of this discussion to the case $D=1$, one can use CFT, where the fields separate into right and left movers.

\section{H Semiclassical regime from the quantum computation}

We may justify this semiclassical logic to arrive at the expectation value for the current in the steady state Eq. (55) by explicitly evaluating the analogue of Eq. (69) with fixed initial boundary condition $\phi\left(t=0, x^{1}=0\right)=0$ (for simplicity). In particular, let us focus on a single momentum mode - i.e., the solution to the equation of motion for a single momentum mode: $\phi(x, 0)=2 \sin \left(p^{1} x\right) \Theta\left(x^{1}\right)$. The dynamics of this problem is completely one dimensional, and so we need only evolve this forward according to the one dimensional Klein-Gordon equation, where the effective mass is simply $\tilde{p}^{2}+m^{2} \equiv M^{2}$. For simplicity, for the remainder of this section we drop the 1 superscripts on $x$ and $p$.

The integral that we need to evaluate is the function which serves as the coefficient of $B_{p}$ in Eq. (69):

$$
\mathcal{B}_{p} \equiv \int_{-\infty}^{\infty} \frac{\mathrm{dq}}{2 \pi}\left(\frac{1}{\mathrm{q}-p-\mathrm{i} \mathbf{0}}-\frac{1}{\mathrm{q}+p-\mathrm{i} \mathbf{0}}\right) \mathrm{e}^{\mathrm{i} \mathrm{q} x}\left(a_{\mathrm{q}, p}^{+} \mathrm{e}^{-\mathrm{i} E_{\mathrm{q}} t}+a_{\mathrm{q}, p}^{-} \mathrm{e}^{\mathrm{i} E_{\mathrm{q}} t}\right)
$$

Let us focus on the limit where $x, t \rightarrow \infty$. In this case, the exponentials oscillate extremely rapidly and we therefore may employ standard tricks to bound such integrals. In particular, let us break the integral over $\mathrm{q}$ into many small regions. Away from $\mathrm{q}= \pm p$, the pole factors 
and $a_{\mathrm{q}, p}^{ \pm}$are slowly varying and may be approximated well by a constant in a neighborhood of $\mathrm{q}=\mathrm{q}_{0}$ of width $2 \delta \mathrm{q}$. The contribution to $\mathcal{B}_{p}$ from this region, from the $a^{+}$term, is given by

$$
\int_{-\delta \mathrm{q}}^{+\delta \mathrm{q}} \frac{\mathrm{d} \delta \mathrm{q}}{2 \pi} \frac{2 p a_{\mathrm{q}_{0}, p}^{+}}{q^{2}-p^{2}} \exp \left[\mathrm{i}\left(\left(x-E_{\mathrm{q}}^{\prime} t\right) \delta \mathrm{q}-\frac{t}{2} E_{\mathrm{q}_{0}}^{\prime \prime} \delta \mathrm{q}^{2}+\cdots\right)\right]
$$

A nearly identical answer holds for the contribution due to $a^{-}$, but with $t \rightarrow-t$. Primes on $E_{\mathrm{q}}$ stand for derivatives. If $x-E_{\mathrm{q}}^{\prime} t \neq 0$, and the ratio $x / t$ is held fixed while $t \rightarrow \infty$, then this integral is bounded from above by

$$
\frac{4 p a_{\mathrm{q}_{0}, p}^{+} \delta \mathrm{q}_{0}}{\pi\left(\mathrm{q}_{0}^{2}-p^{2}\right)} \frac{1}{x-E_{\mathrm{q}}^{\prime} t}
$$

So long as we avoid the poles, and any point where $x-E_{\mathrm{q}}^{\prime} t=0$, then summing over the boxes at each $q_{0}$ gives us a finite integral that is strictly bounded by $1 / t$ and thus vanishes in the limit $t \rightarrow \infty$.

There are two loopholes to the above argument. Let us first focus on a point away from the poles where $x=E_{\mathrm{q}}^{\prime} t$ (note this will always occur only for $|x| \leq t$ ). In this case, we can instead bound Eq. (126) by the saddle point method, and the integral will decay as $t^{-1 / 2}$, which again vanishes as $t \rightarrow \infty$. Evidently, the regions near $\mathrm{q}= \pm p$ are the only dominant contributions to this integral as $t \rightarrow \infty$, with $x / t$ fixed. In this case, we can thus (after some re-shifting of the variable q) approximate the $a^{+}$contributions to $\mathcal{B}_{p}$ (away from points where $x= \pm E_{p}^{\prime} t$ ):

$$
\begin{aligned}
\mathcal{B}_{p} & \approx \int_{-\infty}^{\infty} \frac{\mathrm{dq}}{2 \pi(\mathrm{q}-\mathrm{i} \mathbf{0})}\left(\mathrm{e}^{\mathrm{i}\left(p x-E_{p} t\right)+\mathrm{iq}\left(x-E_{p}^{\prime} t\right)}-\mathrm{e}^{\mathrm{i}\left(-p x-E_{p} t\right)+\mathrm{iq}\left(x+E_{p}^{\prime} t\right)}\right) \\
& =\mathrm{e}^{\mathrm{i}\left(p x-E_{p} t\right)} \Theta\left(x-E_{p}^{\prime} t\right)+\mathrm{e}^{\mathrm{i}\left(-p x-E_{p} t\right)} \Theta\left(x+E_{p}^{\prime} t\right) .
\end{aligned}
$$

The $a^{-}$contributions to $\mathcal{B}_{p}$ vanish, as $a_{p, p}^{-}=0$. The above equation shows that for any ratio of $x / t$ fixed (and not equal exactly to the group velocity of momentum mode $p$ ), at late times momentum mode $p$ is approximately described by its semiclassical dynamics: a pair of waves traveling to the left or right at the classical (group) velocity. This demonstrates that our evaluation of the expectation values of $\left\langle T^{\mu \nu}\right\rangle$ is asymptotically exact as $x^{1}, t \rightarrow \infty$.

\section{Free Dirac fermion results}

We may straightforwardly generalize the analysis of this paper to the case of fermionic models, such as the free massive Dirac fermions. In this case, there is a $\mathrm{U}(1)$ charge, and we may thermalize the reservoirs with temperatures $T_{\mathrm{L}}, T_{\mathrm{R}}$ and chemical potentials $\mu_{\mathrm{L}}, \mu_{\mathrm{R}}$. The results 
for the energy current and the energy density are

$$
\begin{aligned}
& \left\langle T^{01}\right\rangle_{\mathrm{s}}=\frac{\Gamma\left(\frac{d}{2}\right)}{4 \pi^{d / 2+1}(d-1) !} \sum_{\epsilon= \pm} \int_{0}^{\infty} \mathrm{d} p p^{d} \frac{\sinh \left(\frac{\beta_{\mathrm{R}}-\beta_{\mathrm{L}}}{2} E_{p}\right)}{\cosh \left(\frac{\beta_{\mathrm{R}}}{2}\left(E_{p}-\epsilon \mu_{\mathrm{R}}\right)\right) \cosh \left(\frac{\beta_{\mathrm{L}}}{2}\left(E_{p}-\epsilon \mu_{\mathrm{R}}\right)\right)} \\
& =\frac{d \Gamma\left(\frac{d}{2}\right)}{2 \pi^{d / 2+1}} \sum_{\epsilon= \pm}\left(\zeta_{\frac{m}{T_{\mathrm{L}}}}, \frac{\epsilon \mu_{\mathrm{L}}}{T_{\mathrm{L}}}(d+1) T_{\mathrm{L}}^{d+1}-\zeta_{\frac{m}{T_{\mathrm{R}}}}, \frac{\epsilon \mu_{\mathrm{R}}}{T_{\mathrm{R}}}(d+1) T_{\mathrm{R}}^{d+1}\right) \\
& \left\langle T^{00}\right\rangle_{\mathrm{s}}=\frac{\Gamma\left(\frac{d+1}{2}\right)}{4 \pi^{(d+1) / 2}(d-1) !} \sum_{\epsilon= \pm} \int_{0}^{\infty} \mathrm{d} p p^{d-1} E_{p} \frac{\cosh \left(\frac{\beta_{\mathrm{R}}-\beta_{\mathrm{L}}}{2} E_{p}\right)}{\cosh \left(\frac{\beta_{\mathrm{R}}}{2}\left(E_{p}-\epsilon \mu_{\mathrm{R}}\right)\right) \cosh \left(\frac{\beta_{\mathrm{L}}}{2}\left(E_{p}-\epsilon \mu_{\mathrm{R}}\right)\right)} \\
& =\frac{d \Gamma\left(\frac{d+1}{2}\right)}{2 \pi^{(d+1) / 2}} \sum_{\epsilon= \pm}\left(\tilde{\zeta}_{\frac{m}{T_{\mathrm{L}}}, \frac{\epsilon \mu_{\mathrm{L}}}{T_{\mathrm{L}}}}(d+1) T_{\mathrm{L}}^{d+1}+\tilde{\zeta}_{\frac{m}{T_{\mathrm{R}}}, \frac{\epsilon \mu_{\mathrm{R}}}{T_{\mathrm{R}}}}(d+1) T_{\mathrm{R}}^{d+1}\right),
\end{aligned}
$$

where we define the functions

$$
\zeta_{a, b}(d):=\frac{1}{\Gamma(d)} \int_{0}^{\infty} \mathrm{d} p \frac{p^{d-1}}{\mathrm{e}^{\sqrt{p^{2}+a^{2}}-b}+1}, \quad \tilde{\zeta}_{a, b}(d):=\frac{1}{\Gamma(d)} \int_{0}^{\infty} \mathrm{d} p \frac{p^{d-2} \sqrt{p^{2}+a^{2}}}{\mathrm{e}^{\sqrt{p^{2}+a^{2}}-b}+1} .
$$

In the massless limit with zero chemical potential (corresponding to $a=b=0$ ) both functions in Eq. (130) specialize to $\zeta_{0,0}(d)=\tilde{\zeta}_{0,0}(d)=\left(1-2^{1-d}\right) \zeta(d)$, and the result agrees with [51]. In particular, setting $d=1$ and $m=0$, and using $\zeta(2)=\pi^{2} / 6$ with $\Gamma(1 / 2)=\sqrt{\pi}$, we find again that the coefficients of the powers of temperature all specialize to $\pi / 12$ as required for a $1+1$ CFT with central charge $c=1[33,34]$.

We may also define the SCGF for energy transfer, $F(z)$. Thanks to the results of [61], since this is a free model again one may use the extended fluctuation relations, so that (19) still holds. Energy transfer can be interpreted via Poisson processes again, but only at zero chemical potentials $\mu_{\mathrm{L}}=\mu_{\mathrm{R}}=0$. This is the same situation as that which was found in 1+1-dimensional CFT [33, 34]. Here we find the weight

$$
\omega(q)=\left(2^{d-1} \pi^{\frac{d+1}{2}} \Gamma\left(\frac{d+1}{2}\right)\right)^{-1} \sum_{n=1}^{[|q| / m]} \frac{(-1)^{n-1}}{n^{d+1}}\left(q^{2}-n^{2} m^{2}\right)^{\frac{d-1}{2}} \cdot \begin{cases}\mathrm{e}^{-\beta_{\mathrm{L}} q} & (q>0) \\ \mathrm{e}^{\beta_{\mathrm{R}} q} & (q<0)\end{cases}
$$

\section{References}

[1] G. Gallavotti and E. G. D. Cohen, "Dynamical ensembles in nonequilibrium statistical mechanics", Phys. Rev. Lett. 74, 2694 (1995).

[2] C. Jarzynski and D. K. Wojcik, "Classical and quantum fluctuation theorems for heat exchange", Phys. Rev. Lett. 92, 230602 (2004), arXiv: cond-mat/0404475.

[3] M. Esposito, U. Harbola and S. Mukamel, "Nonequilibrium fluctuations, fluctuation theorems and counting statistics in quantum systems", Rev. Mod. Phys. 81, 1665 (2009), arXiv:0811.3717.

[4] C. Caroli, R. Combescot, P. Nozieres and D. Saint-James, "Direct calculation of the tunneling current", J. Phys. C4, 916 (1971). 
[5] R. J. Rubin and W. L. Greer, "Abnormal lattice thermal conductivity of a one-dimensional, harmonic, isotopically disordered crystal", J. Math. Phys. 12, 1686 (1971).

[6] H. Spohn and J. L. Lebowitz, "Stationary Non-Equilibrium States of Infinite Harmonic Systems", Commun. Math. Phys. 54, 97 (1977).

[7] S. Tasaki, "Nonequilibrium stationary states of nonintercting electrons in a one-dimensional lattice", Chaos, Solitons and Fractals 12, 2657 (2001)

[8] S. Tasaki, "Nonequilibrium stationary states for a quantum 1-d conductor", AIP Conf. Proc. 519, 356 (2000).

[9] H. Araki and T. G. Ho: "Asymptotic time evolution of a partitioned infinite two-sided isotropic XY- chain", Proc. Steklov Inst. Math. 228, 203 (2000).

[10] Y. Ogata, "Nonequilibrium properties in the transverse XX chain", Phys. Rev. E66, 016135 (2002).

[11] W. H. Aschbacher and C-A. Pillet, "Non-equilibrium steady states of the XY chain", J. Stat. Phys. 112, 1153 (2003).

[12] D. Ruelle, "Natural nonequilibrium states in quantum statistical mechanics", J. Stat. Phys. 98, 57 (2000), arXiv:math-ph/9906005.

[13] H.D. Cornean, V. Moldoveanu and C.-A. Pillet, "On the steady state correlation functions of open interacting systems", Commun. Math. Phys. 331265 (2014), arXiv: 1305. 4410.

[14] J. Rammer and H. Smith, "Quantum field-theoretical methods in transport theory of metals", Rev. Mod. Phys. 58, 323 (1986).

[15] Y. Meir and N. S. Wingreen, "Landauer formula for the current through an interacting electron region", Phys. Rev. Lett. 68, 2512 (1992).

[16] S. Hershfield, "Reformulation of steady state nonequilibrium quantum statistical mechanics", Phys. Rev. Lett. 70, 2134 (1993).

[17] A.-P. Jauho, N. S. Wingreen and Y. Meir., "Time-dependent transport in interacting and noninteracting resonant-tunneling systems", Phys. Rev. B 50, 5528 (1994), arXiv: cond-mat/9404027.

[18] B. Doyon and N. Andrei, "Universal aspects of nonequilibrium currents in a quantum dot", Phys. Rev. B73, 245326 (2006), arXiv: cond-mat/0506235.

[19] B. Doyon, "New method for studying steady states in quantum impurity problems: the interacting resonant level model", Phys. Rev. Lett. 99, 076806 (2007), arXiv: cond-mat/0703249.

[20] L. Freton and E. Boulat, "Out-of-equilibrium properties and non-linear effects for interacting quantum impurity systems in their strong coupling regime", Phys. Rev. Lett. 112, 216802 (2014), arXiv: 1303.7441.

[21] P. Fendley, A. W. W. Ludwig and H. Saleur, "Exact conductance through point contacts in the $\nu=1 / 3$ fractional quantum hall effect", Phys. Rev. Lett. 74, 3005 (1995), arXiv: cond-mat/9408068. 
[22] P. Fendley, A. W. W. Ludwig and H. Saleur, "Exact nonequilibrium transport through point contacts in quantum wires and fractional quantum Hall devices", Phys. Rev. B52, 8934 (1995), arXiv: cond-mat/9503172.

[23] E. Boulat, H. Saleur, and P. Schmitteckert, "Twofold advance in the theoretical understanding of far-from-equilibrium properties of interacting nanostructures", Phys. Rev. Lett. 101, 140601 (2008), arXiv:0806.3731

[24] A. Komnik and H. Saleur, "Quantum fluctuation theorem in an interacting setup: point contacts in fractional quantum hall edge state devices", Phys. Rev. Lett. 107, 100601 (2011), arXiv:1109.3874.

[25] S. T. Carr, D. A. Bagrets and P. Schmitteckert, "Full counting statistics in the self-dual interacting resonant level model", Phys. Rev. Lett. 107, 206801 (2011), arXiv:1104.3532.

[26] L. S. Levitov and G. B. Lesovik, "Charge distribution in quantum shot noise", JETP Lett. 58, 230 (1993), arXiv:cond-mat/9401004.

[27] I. Klich, "An elementary derivation of Levitov's formula. A short review," in Quantum Noise in Mesoscopic Physics, NATO Science Series III, ed. Yu. Nazarov, 397 (Kluwer, 2003), arXiv: cond-mat/0209642.

[28] K. Schönhamer, "Full counting statistics for non-interacting fermions: Exact results and the Levitov-Lesovik formula," Phys. Rev. B75, 205329 (2007) arXiv:cond-mat/0701620.

[29] J. E. Avron, S. Bachmann, G. M. Graf, and I. Klich, "Fredholm determinants and the statistics of charge transport," Commun. Math. Phys. 280, 807 (2008), arXiv:0705.0099.

[30] D. Bernard and B. Doyon, "Full counting statistics in the resonant-level model", J. Math. Phys. 53, 122302 (2012), arXiv:1105.1695..

[31] D. B. Gutman, Y. Gefen and A. D. Mirlin, "Bosonization out of equilibrium", Europhys. Lett. 90, 37003 (2010), arXiv:0911.4559.

[32] D. B. Gutman, Y. Gefen and A. D. Mirlin, "Full counting statistics of Luttinger liquid conductor", Phys. Rev. Lett. 105, 256802 (2010), arXiv:1003.5433.

[33] D. Bernard and B. Doyon, "Energy flow in non-equilibrium conformal field theory", J. Phys. A45, 362001 (2012), arXiv:1202.0239.

[34] D. Bernard and B. Doyon, "Non-equilibrium steady-states in conformal field theory", Ann. Henri Poincaré (2014), arXiv:1302.3125.

[35] O. Castro-Alvaredo, Y. Chen, B. Doyon and M. Hoogeveen, "Thermodynamic Bethe ansatz for non-equilibrium steady states: exact energy current and fluctuations in integrable QFT", J. Stat. Mech. P03011 (2014), arXiv:1310.4779.

[36] B. Doyon, "Nonequilibrium density matrix for thermal transport in quantum field theory", arXiv: 1212.1077.

[37] A. De Luca, J. Viti, D. Bernard and B. Doyon, "Non-equilibrium thermal transport in the quantum Ising chain", Phys. Rev. B88, 134301 (2013), arXiv:1305. 4984. 
[38] C. Karrasch R. Ilan and J. E. Moore, "Nonequilibrium thermal transport and its relation to linear response", Phys. Rev. B88, 195129 (2013), arXiv:1211.2236.

[39] C. Karrasch, J. H. Bardarson and J. E. Moore, "Reducing the numerical effort of finitetemperature density matrix renormalization group transport calculations", New. J. Phys. 15, 083031 (2013), arXiv: 1303.3942.

[40] M. J. Bhaseen, B. Doyon, A. Lucas and K. Schalm, "Far from equilibrium energy flow in quantum critical systems", arXiv:1311.3655.

[41] Z. Rieder, J. L. Lebowitz, and E. Lieb, "Properties of a harmonic crystal in a stationary nonequilibrium state", J. Math. Phys. 8, 1073 (1967).

[42] H. Nakazawa, "Energy flow in harmonic linear chain", Prog. Theor. Phys. 39, 236 (1968)

[43] H. Nakazawa, "On the lattice thermal conduction", Prog. of Theor. Phys. Suppl. 45, 231 (1970).

[44] U. Zurcher and P. Talkner, "Quantum-mechanical harmonic chain attached to heat baths. II. Nonequilibrium properties", Phys. Rev. A42, 3278 (1990).

[45] K. Saito, S. Takesue and S. Miyashita, "Energy transport in the integrable system in contact with various types of phonon reservoirs", Phys Rev E61, 2397 (2000), arXiv: cond-mat/9810069.

[46] D. Segal, A. Nitzan and P. Hänggi, "Thermal conductance through molecular wires", J. Chem. Phys. 119, 6840 (2003), arXiv:physics/0306187

[47] A. Dhar and D. Roy, "Heat transport in harmonic lattices", J. Stat. Phys. 125, 801 (2006), arXiv: cond-mat/0606465.

[48] K. Saito and A. Dhar, "Fluctuation theorem in quantum heat conduction", Phys. Rev. Lett. 99, 180601 (2007), arXiv: cond-mat/0703777.

[49] D. Roy and A. Dhar, "Heat transport in ordered harmonic lattices", J. Stat. Phys. 131, 535 (2008), arXiv:0711.4318.

[50] A. Dhar, K. Saito, P. Hänggi, "Nonequilibrium density matrix description of steady state quantum transport", Phys. Rev. E85, 011126 (2012), arXiv:1106.3207.

[51] M. Collura and G. Martelloni, "Non-equilibrium transport in $d$-dimensional non-interacting Fermi gases", J. Stat. Mech. P08006 (2014), arXiv:1404.1319.

[52] P. Mazur, "Non-ergodicity of phase functions in certain systems", Physica 43, 533 (1969).

[53] X. Zotos, F. Naef, and P. Prelovsek, "Transport and conservation laws", Phys. Rev. B55, 11029 (1997).

[54] M. Q. Zhang, J.-S. Wang, J. L. Lebowitz and J. L. Vallés, "Power law decay of correlations in stationary nonequilibrium lattice gases with conservative dynamics," J. Stat. Phys. 52, 1461 (1988).

[55] J. R. Dorfman, T. R. Kirkpatrick, and J. V. Sengers, "Generic long-range correlations in molecular fluids", Ann. Rev. of Phys. Chem. 45, 213 (1994). 
[56] B. Doyon, "The density matrix for quantum impurities out of equilibrium", Lecture notes for the Fifth Capri Spring School on Transport in Nanostructures (2009), Quantumimpurities.pdf.

[57] M. Mierzejewski, P. Prelovšek, and T. Prosen, "Breakdown of the generalized Gibbs ensemble for current generating quenches", Phys. Rev. Lett. 113020602 (2014), arXiv:1405.2557.

[58] W. H. Aschbacher and J.-M. Barbaroux, "Out of equilibrium correlations in the XY chain", Lett. Math. Phys. 77, 11 (2006), arXiv:math-ph/0505062.

[59] Y. Chen and B. Doyon, "Form factors in equilibrium and non-equilibrium mixed states of the Ising model", J. Stat. Mech (to appear), arXiv:1305.0518.

[60] M. Collura and D. Karevski, "Quantum quench from a thermal tensor state: boundary effects and generalized Gibbs ensemble", Phys. Rev. B89 214308 (2014), arXiv:1402.1944.

[61] D. Bernard and B. Doyon, "Time-reversal symmetry and fluctuation relations in nonequilibrium quantum steady states", J. Phys. A46, 372001 (2013), arXiv:1306. 3900. 